\title{
Z-Area Saltstone Disposal Facility Groundwater Monitoring Report (1998 Annual Report)
}

by

D. Wells

RECORDS ADMINISTRATION

Westinghouse Savannah River Company

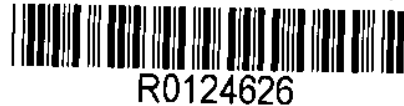

Savannah River Site

Aiken, South Carolina 29808

DOE Contract No. DE-AC09-96SR18500

This paper was prepared in connection with work done under the above contract number with the U. S.

Department of Energy. By acceptance of this paper, the publisher and/or recipient acknowledges the U.S. Government's right to retain a nonexclusive, royalty-free license in and to any copyright covering this paper, along with the right to reproduce and to authorize others to reproduce all or part of the copyrighted paper. 
WSRC-RP-99-00002

Unclassified

\section{Z-AREA SALTSTONE DISPOSAL FACILITY GROUNDWATER MONITORING REPORT (U) \\ 1998 ANNUAL REPORT January 1999}

Authorized Derivituive Classifier And Reyiewing Official:

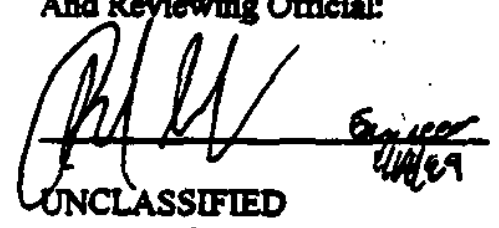

Does Not Contain Unciassified Controlled Nuclear Information 


\section{DISCLAIMER}

This report was prepared as an account of work sponsored by an agency of the United States Government. Neither the United States Government nor any agency thereof, nor any of their employees, makes any warranty, express or implied, or assumes any legal liability or responsibility for the accuracy, completeness, or usefulness of any information, apparatus, product, or process disclosed, or represents that its use would not infringe privately owned rights. Reference herein to any specific commercial product, process, or service by trade name, trademark, manufacturer, or otherwise does not necessarily constitute or imply its endorsement, recommendation, or favoring by the United States Government or any agency thereof. The views and opinions of authors expressed herein do not necessarily state or reflect those of the United States Govemment or any agency thereof.

This report has been reproduced directly from the best available copy.

Available to DOE and DOE contractors from the Office of Scientific and Technical Information, P.O. Box 62, Oak Ridge, TN 37831; prices available from (615) 576-8401.

Available to the public from the National Technical Information Service, U.S. Department of Commerce, 5285 Port Royal Road, Springfield, VA 22161. 


\section{INTRODUCTION}

In accordance with SRS Z-Area Saltstone Industrial Solid Waste Permit, \#025500-1603, wells ZBG-1, ZBG-1A and ZBG-2 are monitored for the parameters listed in Table 1.

Sampling was done during the first and third quarters of 1998. Additional analyses were also run. The analytical results appear in Appendix I.

The well sampling and analyses were conducted in accordance with Procedure Manual 3Q5, Hydrogeologic Data Collection. Well ZBG-1A, which is screened in a perched water zone, was dry during third quarter and could not be sampled. This is not unusual.

The sampling did not reveal any evidence of a release from the Saltstone vauits. Flow directions and velocities were similar to those observed in past years.

\section{DISCUSSION}

\section{Flow Direction and Rate}

The uppermost true aquifer beneath Z-Area is monitored by wells ZBG-1 and ZBG-2. Water level measurements from these wells were used along with water levels from nearby areas to construct potentiometric maps for first and third quarters (figures 1 and 2). Recent reductions in groundwater monitoring at SRS have resuited in a scarcity of water level data in 1998 compared to past years. Beginning in 1999 we will assure that water levels are taken in S-Area and Y-Area 80 that we can construct more dependable maps.

But even with relatively scarce data it is possible to construct sensible potentiometric maps. This is because McQueen's Branch and Upper Three Runs Creek are the obvious discharge points for shallow groundwater in the area and, hence, provide excellent control. The two streams meet just north of Z-Area. The flow directions shown on the 1998 maps are similar to those shown in previous years.

Flow rate can be estimated using the following equation:

$$
\text { Flow }(\mathrm{ft} / \mathrm{day})=\frac{\text { Hydraulic Conductivity (ft/day) }}{\text { Porosity (unitless) }} \times \frac{\mathrm{dh}(\mathrm{ft})}{\mathrm{dl}(\mathrm{ft})}
$$

Where the hydraulic conductivity constant is $1.7 \mathrm{ft} /$ day, the effective porosity value is 30 percent, the change in head is $\mathrm{dh}$, and the horizontal distance along each flow arrow is $\mathrm{dl}$.

The site's Geographical Information System (GIS) was used to determine the flow path length in meters. This was converted to feet and used to calculate the flow rate. For first quarter of 1998, the calculation is as follows:

$\underline{1.7} \times \underline{7.5}=0.023 \mathrm{ft} / \mathrm{day}$ or $12 \mathrm{ft} /$ year

$0.30 \quad 1300$

For third quarter of 1998 , the calculation is as follows:

$\underline{1.7} \times \underline{11.6}=0.036 \mathrm{ft} /$ day or $13 \mathrm{ft} /$ year

$0.30 \quad \frac{11.6}{1860}$ 


\section{Analytical results}

Six analytes were detected in downgradient well ZBG-2: gross alpha, gross beta, barium, nitrate/nitrite, mercury, and radium 226. None of these detections represent evidence of a release from the vaults.

The maximum gross alpha result for ZBG-2 was $1.22 \mathrm{pCi} / \mathrm{L}$ from the 8-4-98 sample. This is lower than the result from upgradient well ZBG-1 (1.85 pCi/L). It is also lower than the nonparametric upper tolerance limit $(6.0 \mathrm{pCi} / \mathrm{L})$ estabished using historic data.

The maximum gross beta result from ZBG.2 was $1.45 \mathrm{pCi} / \mathrm{L}$ from the 8-4-98 sample. This is lower than the result from the upgradient well $(4.47 \mathrm{pCi} / \mathrm{L})$. It is also well below the nonparametric upper tolerance limit of $13.4 \mathrm{pCi} / \mathrm{L}$.

The maximum result for radium 226 was $1.67 \mathrm{pCi} / \mathrm{L}$. This is slightly higher than the upgradient result for the same day $(0.59 \mathrm{pCi} / \mathrm{L})$. However, it is well below the nonparametric upper tolerance limit of $11 \mathrm{pCi} / \mathrm{L}$. It should also be noted that as recently as 2-2-98, the background well yielded a result of $7.78 \mathrm{pCi}$.

Barium has been detected pretty consistently in the ZBG wells. Historical data from upgradient well ZBG-1 have been used to calculate an upper tolerance limit of $10.4 \mathrm{ug} / \mathrm{L}$. Results from ZBG-2 were below this limit during both sampling periods of 1998 . During both periods, the ZBG-2 results were lower than the ZBG-1 results ( $6.1 \mathrm{ug} / \mathrm{L}$ vs. $9.9 \mathrm{mg} / \mathrm{L}$ in February and $6.4 \mathrm{mg} / \mathrm{L}$ v9. $9.7 \mathrm{mg} / \mathrm{L}$ in August).

As with barium, the ZBG-2 results for nitrate-nitrite were below the calculated tolerance limit of $1886.9 \mathrm{ug} / \mathrm{L}$. They were also below ZBG-1 results from samples taken at the same time. In February ZBG-2 yielded a result of $79.0 \mathrm{ug} / \mathrm{L}$ compared to $188 \mathrm{ug} / \mathrm{L}$ from $\mathrm{ZBG}-1$. In August the ZBG-2 result was $560 \mathrm{ug} / \mathrm{L}$ compared to $1360 \mathrm{ug} / \mathrm{L}$ in $\mathrm{ZBG}-1$.

The mercury result reported for the 8.4-98 sample from ZBG-2 is .7 ppb. This appears to be a reporting error. More often than not, $.7 \mathrm{ppb}$ is reported as the detection limit for mercury (evidenced by a " $U$ " in the qualifier column). In this case, no " $U$ " is recorded indicating a one time detection of exactly .7 ppb. It is very likely that the " $U$ " was accidentally omitted and that this sample was actually below detection for mercury. It should be noted that the duplicate sample was below detection. 


\section{DETECTION MONITORING PARAMETERS}

pH (field \& lab)

Specific Conductance (field)

Water level in M.S.L. (tenth/feet)

Arsanic

Antimony

Barium

Cadmium

Chromium

Lead

Mercuny.

Nitrate (as Nitrogen)

Nitrite (as Nitrogen)

Selonium

Silver

Benzene and Toluene

Radionuclides

-Radium 226 and 228 (sum)

-Gross alpha particle ectivity.

- Beta particle and proton radioactivity

\section{Analyses of the metals should be performed on unfiltered groundwater samples.}

Table 1. Detection monitoring parameters (from permit $\$ 025500-1603$ ). 


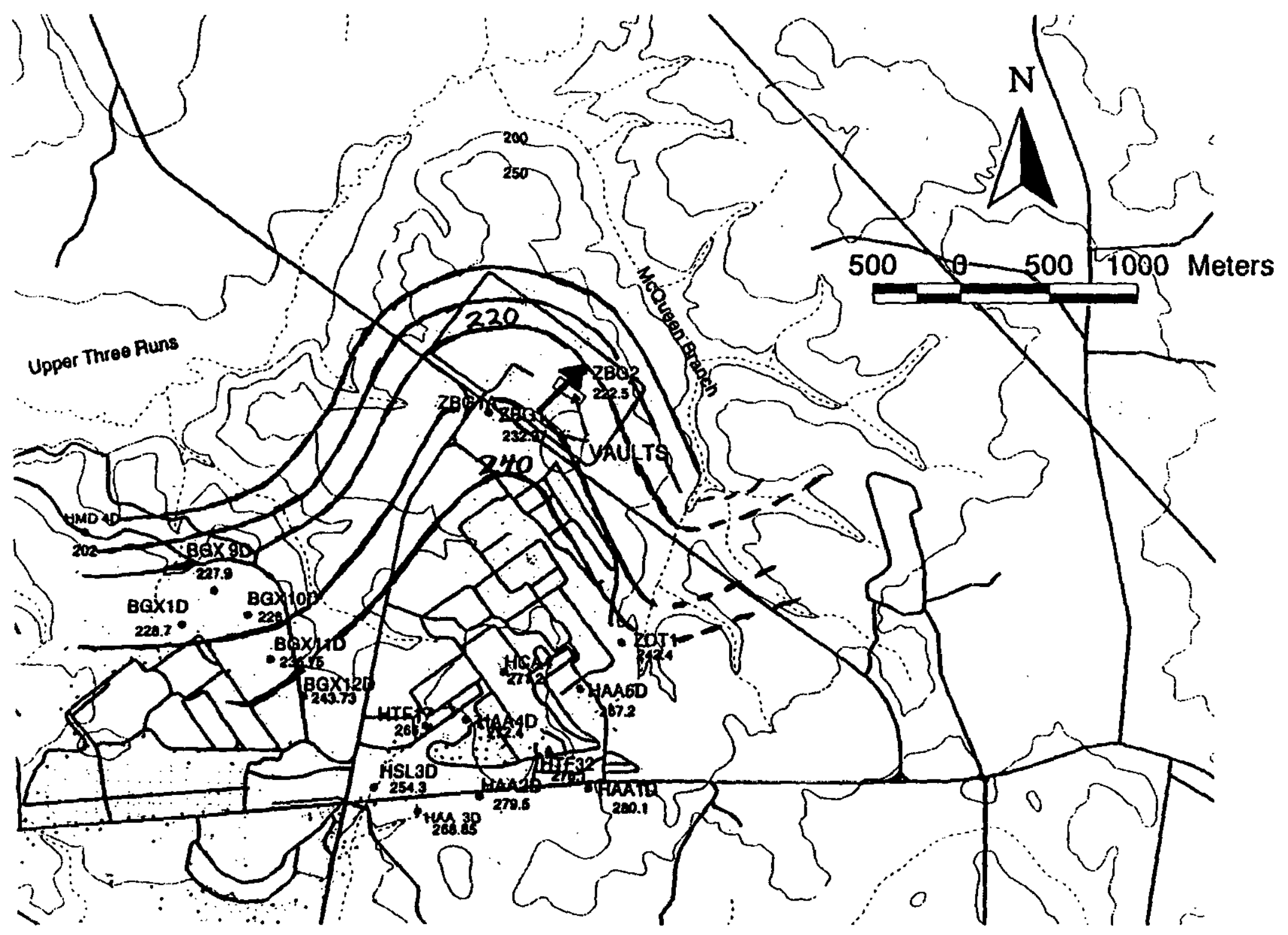

Figure 1. Potentiometric surface of water table aquifer- First Quarter, 1998. 


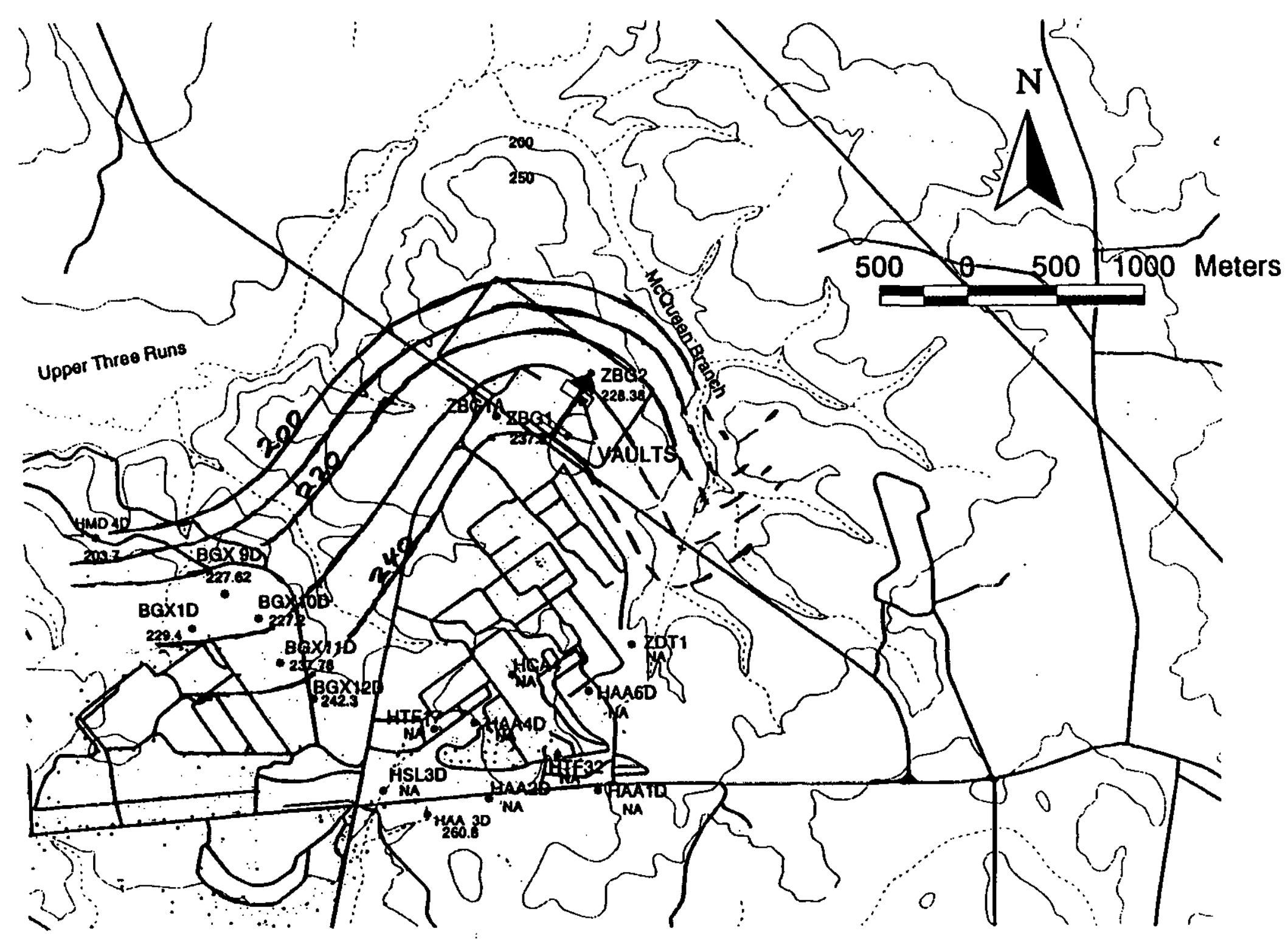

Figure 2. Potentiometric surface of water table aquifer- Third Quarter, 1998. 
APPENDIX I

ANALYTICAL DATA 


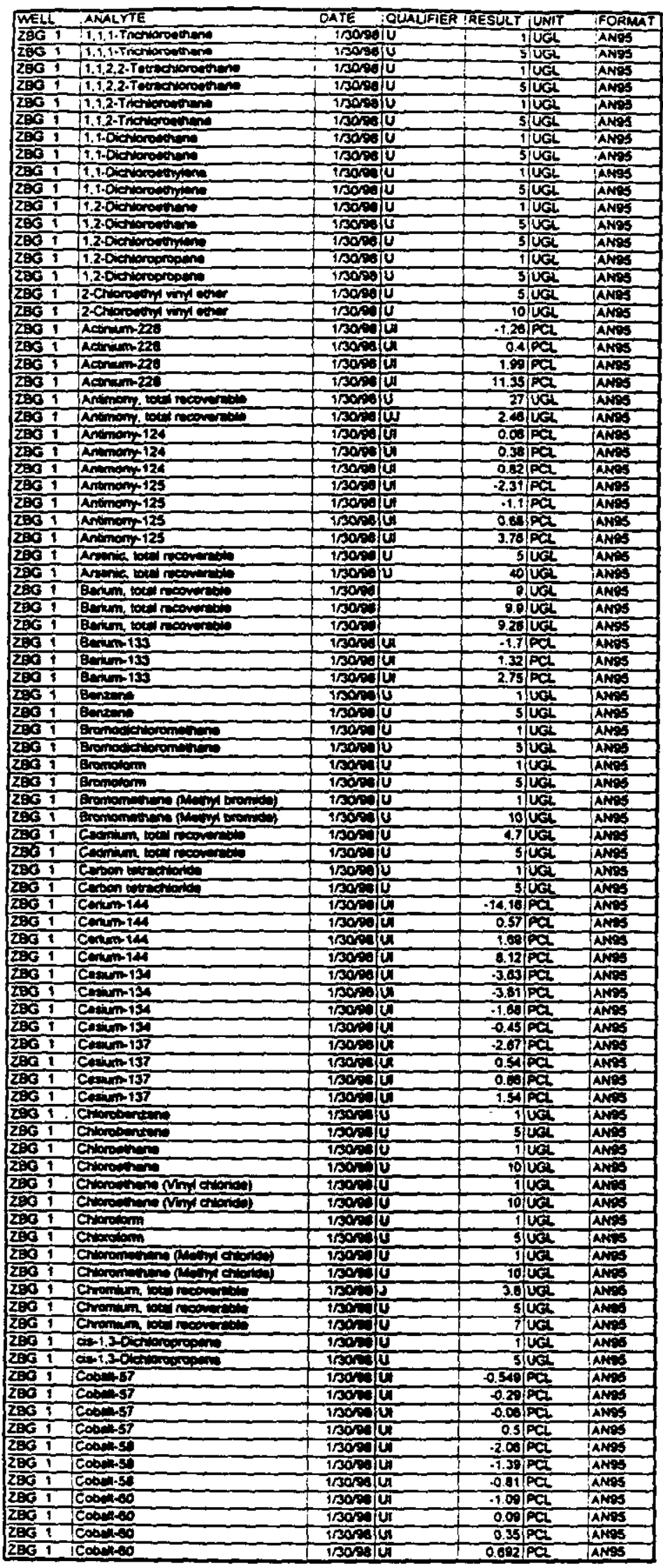

WSRC-RP.99-00002 


\begin{tabular}{|c|c|c|c|c|c|c|}
\hline WEL & ANALITE & DATE & OUAUFER & RESTULT & UNIT & FORMAT \\
\hline 2861 & Dioremegrioromentem & 1 300s & IU. & & IIUGL & IANOS \\
\hline ZEG 1 & 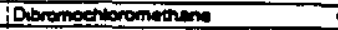 & $1 / 3000$ & iv & & 5:UG & ANis \\
\hline$\overline{2 E G 9}$ & 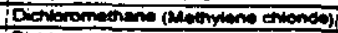 & i1 130000 & u & 3.34 & UTL & Inos \\
\hline ZEG 1 & 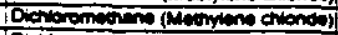 & $1 / 30 \mathrm{sa}$ & U & 1.84 & AUE & TaNBS \\
\hline EEC 1 & 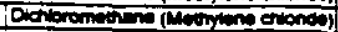 & $130 \mathrm{as}$ & Iu & 5.24 & UUE & ANDS \\
\hline 2861 & Etroonxane & 1r30\%: & Iu & & TUGL & ANos \\
\hline$\angle E G T$ & 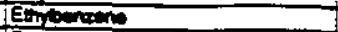 & 1500 & ú & & SIUGL & ANOS \\
\hline 2861 & Eucon 152 & 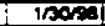 & Iิ & .1 .71 & 1]PCL & AnOS \\
\hline 2861 & Eurominis & 1300 & wi & 0.490 & DPCl & taves \\
\hline ZEG 1 & Europien-152 & $130 \times 3$ & Uि & 12.62 & |PCL & ANSS \\
\hline $28 \overline{1} 1$ & Euroginimis 15 & 1300is & IUI & 13.55 & 5 PCL & Anes \\
\hline 2001 & 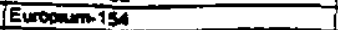 & $1 / 30 \mathrm{ar}$ & IUt & -2.92 & $3 \mid \mathrm{PCL}$ & Amis \\
\hline 2861 & Eurecin-1S & $1 / \operatorname{son}$ & $\overline{\mathbf{u}}$ & -0.904 & $A P C L$ & ANES \\
\hline 2861 & Eurooumis & 1300 & 녀 & & 2म्CL & Anes \\
\hline 286 I & Evopinim-15s & $1 / 30 \%$ & UI & 3.33 & अव & ANAS \\
\hline ZEG 1 & Europinm 1ss & 1/300/ & UI & -2.29 & IDCL & ANis \\
\hline 2801 & Europentiss & 130: & UT & 2.00 & $P Q$ & ANDS \\
\hline $2 B 61$ & Europin-153 & 1sond & UH & 2.4 & PCL & ANOS \\
\hline 2801 & Expopin-15s & Iromi & IU & 4.53 & IPCL & Anes \\
\hline EEO 1 & Groal erome & $1 / 300$ & & 1.29 & बिC & ANS \\
\hline ZEG 1 & Gous clope & 1/300 & & 2.33 & $\overline{P C}$ & ANS \\
\hline 2801 & Grous ating & 130\% & & 2.5 & PCL & ANes \\
\hline ZeG 1 & Grosenten & $1 / 30 \mathrm{~s}$ & & 3.42 & $\sqrt[P C]{ }$ & ANBS \\
\hline 2001 & 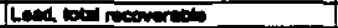 & $1 / 3000$ & $\sqrt{J}$ & 7.3 & Uूa & ANS \\
\hline 2961 & 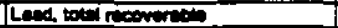 & $1 / 3007$ & J & 7.5 & SUदL & ANES \\
\hline 2801 & 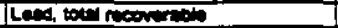 & $1 / 3000$ & U & 6.46 & Uिद्ध & ANis \\
\hline $2 E G 1$ & Laed-212 & 1/3000 & UI & -1.97 & $\sqrt{A C L}$ & ANBS \\
\hline 2069 & $50 \cos 212$ & $1 / 3000$ & UT & 0.12 & ACL & Ans \\
\hline 2801 & Leec212 & $1 / 30 \mathrm{rat}$ & पiv & 1.85 & $\overline{P C L}$ & ANS \\
\hline 2001 & Lend212 & $1 / 3002$ & Ui & 5.49 & PCL & ANS \\
\hline 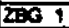 & Menamians & 1/300: & $\bar{\omega}$ & .1 .04 & PCL & ans \\
\hline 2001 & 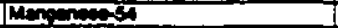 & 130ia & $\overline{\mathbf{T}}$ & 0.11 & FCL & ANG \\
\hline ZEO 1 & Mnsing & 1/300: & $\mathbf{U}$ & 0.4 & ECI & Ans \\
\hline 2001 & 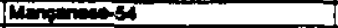 & T/30; & $\mathbf{\omega}$ & 1.54 & AC & AN:S \\
\hline ZBOA & 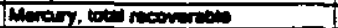 & trast & $\bar{U}$ & 0.2 & Uat & ANis \\
\hline $2 B O 1$ & 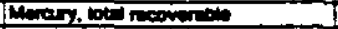 & 1300 & 0 & 0.7 & Ua्2 & axis \\
\hline ZEG I & 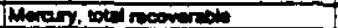 & 130 & $\bar{U}$ & & Uिद्र & ANS \\
\hline 2801 & 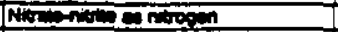 & 1/3001 & & $15 \mathrm{~A}$ & Ua & ANDS \\
\hline EQB & Ain & 1/sari & & 180 & IUCL & Anis \\
\hline 2801 & 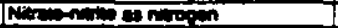 & 1r3060 & & 1350 & Juch & ANes \\
\hline 2801 & Nom an nion & rsont & W & 20 & tuan & ANDS \\
\hline 2001 & Nimenten & $130 \mathrm{n}$ & W & 50 & IUa & ANes \\
\hline 2001 & Nomolnow & $130 \mathrm{Di}$ & $\sqrt{5}$ & 3.30 & अव & ANis \\
\hline 2001 & Nomolen best & 1305 & & 4.10 & SIPC & ANBS \\
\hline 2801 & 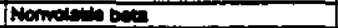 & 1/30r| & I & 9.39 & Pिa & ANCS \\
\hline 2001 & 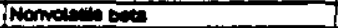 & 1/300 & & 5.22 & $\sqrt{P C}$ & ANSS \\
\hline 2001 & Int & 1300 & 5 & 5.14 & PM & ANDS \\
\hline 2001 & iph & 130\% & 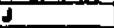 & 5.22 & FW & laves \\
\hline 2861 & $\mathrm{Ign}$ & $130 \mathrm{D}$ & $J$ & 5.53 & 3 & ANis \\
\hline 2801 & $p$ & $1 / 30 / 2]$ & J & 5.9 & DW & NA:S \\
\hline 2801 & 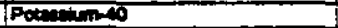 & 13001 & $\overline{\mathbf{U}}$ & .12 .00 & $\overline{P C}$ & ANis \\
\hline 2801 & Potescing-10 & 13000 & $\bar{\omega}$ & 12.4 & PCa & aros \\
\hline ZEG 1 & Poracion-o & $1 / 30 \%$ & $\bar{u}$ & 19.37 & $\sqrt{P C}$ & $A \times 3$ \\
\hline $2: 61$ & Petatsones & 1000 & $\bar{U}$ & 42.54 & ACa & $A \div 3$ \\
\hline $2: 61$ & Promectionit4 & $130 \%$ & $\overline{\mathbf{u}}$ & -2.75 & $5 \overline{P C L}$ & $A$ \\
\hline 2694 & Promentinging & $130 \mathrm{pol}$ & U: & 0.35 & FCL & $A+3$ \\
\hline 2001 & 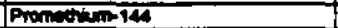 & $\sin 2$ & is & 0.501 & $\sqrt{P C}$ & $A \times B$ \\
\hline$\angle D O 1$ & Promering 144 & Trope & $\mathbf{O}$ & 4.50 & PCL & ANOB \\
\hline ZEG 1 & Promeninen 160 & 1300 & (i) & 6.05 & $\sqrt{F C}$ & ANS \\
\hline$\angle \mathrm{BO} 1$ & Prome - & tracid & U & 0.85 & PCQ & AN:S \\
\hline 2801 & Pros & 1/3000] & $\mathbf{U}$ & 323 & Bक्a & ANis \\
\hline $2 \mathrm{BOC} 1$ & Prome & 1300i & U & 4.15 & SFE & ANS \\
\hline $2 \mathrm{EO} 1$ & 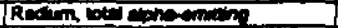 & 1row & 0 & 0.23 & 3PC & ANis \\
\hline ZEO 1 & 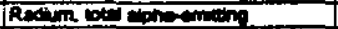 & $150 \mathrm{at}$ & U & 0.01 & IRa & ANES \\
\hline $2 B{ }_{1}$ & 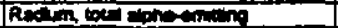 & 150 & & 0.20 & $\sqrt{P C}$ & $\sqrt{\text { N.S }}$ \\
\hline 2801 & 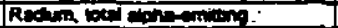 & $1 / 305$ & & 0.6 & FCL & tâis \\
\hline $2 E B 1$ & 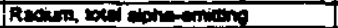 & 1sont & & 0.7 & Pa & AN:S \\
\hline 401 & 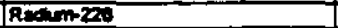 & 1300 & $J$ & 0.07 & FCL & AN:S \\
\hline ZEG 1 & Redienta: & $150+1$ & 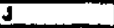 & 0.00 & JPC & Ans \\
\hline $2 \overline{Z E 1}$ & 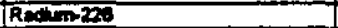 & 150it & U. & 0.354 & FC & ANis \\
\hline 2801 & 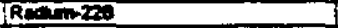 & 1500 il & $\mathbf{L}$ & 0.430 & FCL & Nois \\
\hline 2801 & Ren 20 & 130011 & $\bar{U}$ & 0.25 & $\overline{P C A}$ & Axis \\
\hline 2801 & Aacinas & 1000 & $\mathbf{U}$ & 0.00 & BC2 & AN. \\
\hline 2801 & Rechen & 100 & & 4.28 & BलC & ANS \\
\hline 2861 & Ruthenimios & $1 \mathrm{sen}$ & U. & .20 .30 & IPC & $M=8$ \\
\hline 2001 & Ruthengos & 150it & U. & -4.22 & 3्FC2 & NAS \\
\hline ZEG 1 & Ruereamias & $130=$ - & $\bar{\omega}$ & 9.11 & IfCa & $\operatorname{Nos}$ \\
\hline 2961 & 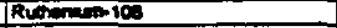 & $100 \mathrm{D}$ & $\mathbf{\omega}$ & 30.76 & FC & ans \\
\hline 2001 & 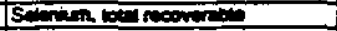 & 100 & 5 & 1.95 & पिa & ANis \\
\hline 2831 & 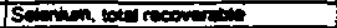 & 150001 & (0) & $\infty$ & Uिद & Tanis \\
\hline 2001 & 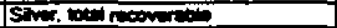 & 1300: & & 0.74 & Uuet & AN:S \\
\hline 2801 & 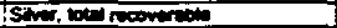 & 1/300: & $\sqrt{J}$ & 0.024 & Uिa & ANS \\
\hline 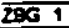 & 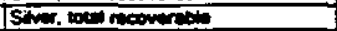 & 1/300:0 & $\mathbf{U}$ & & iva & ANSS \\
\hline 2001 & Sodim 22 & tr30\%int & $\bar{U}$ & -0.04 & FCA & ANDS \\
\hline $28 O_{1}$ & Sochm-22 & Tsowit & Uu & 0.341 & 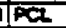 & ANis \\
\hline 286 & Sodem22 & $1 / 30 \%$ & W & 0.34 & IACL & Anis \\
\hline 2801 & $500 \mathrm{~m}-22$ & 130\% & $\mathbf{U}$ & 0.61 & PCa & ANSS \\
\hline ZEG 1 & Teconouin-es & $1 / 30 \%$ & Ü & $\mathbf{4 . 6 1}$ & IPCL & TANSS \\
\hline
\end{tabular}

WSRC-RP-99-00002 


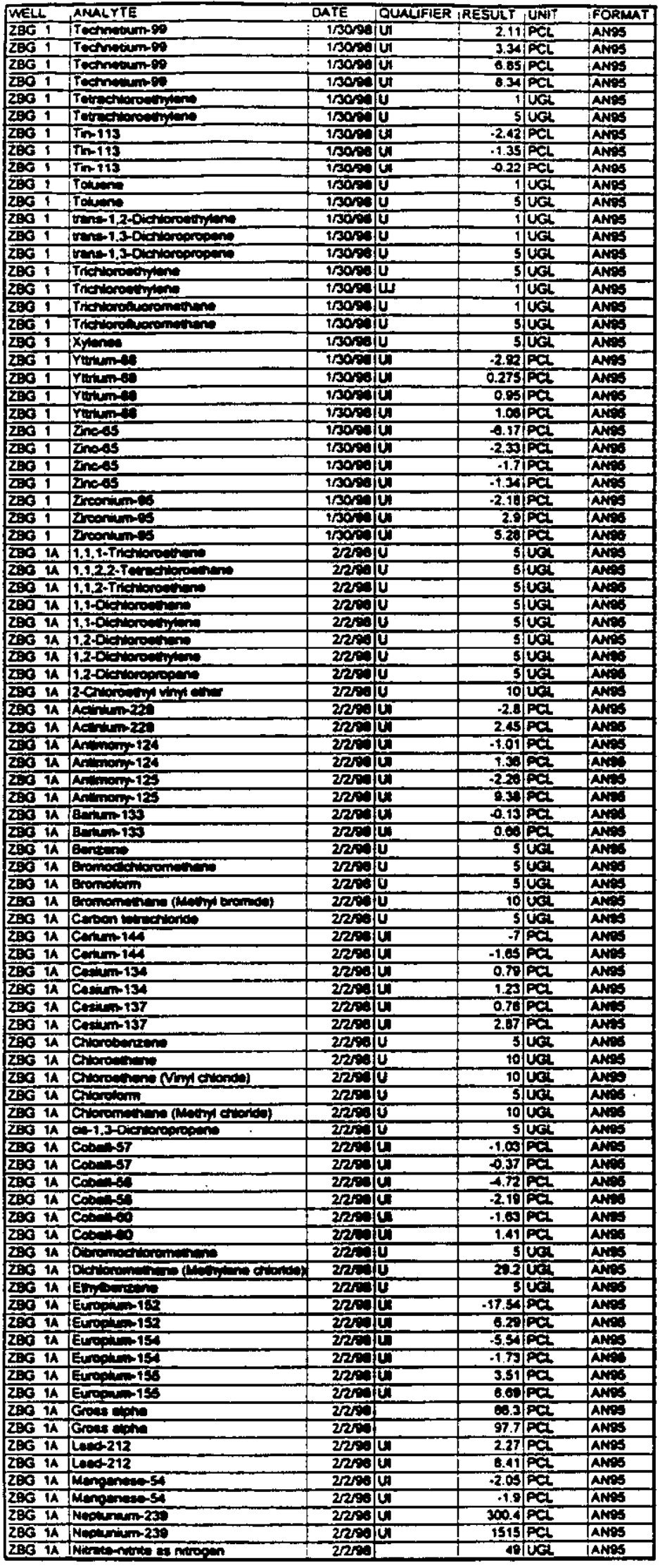

WSRC-RP-99-00002 


\begin{tabular}{|c|c|c|c|c|c|}
\hline WELL & IANALYTE & DATE & QUALFIER & JRESULT UNAT & FOARAT \\
\hline 28614 & inveros as nitroen & $22 \pi 0$ & w & 2 UaL & ANDS \\
\hline $280 \mathrm{TA}$ & Nomeletio ber & $2 \sqrt{250}$ & & $41 . \overline{P A C C}$ & ANOS \\
\hline ZEG TA & Nonnolen bee & $2 \sqrt{200}$ & & 52.561PC & ANDS \\
\hline $2 B G 9$ & IDH & 2द्या & J & $8.261 \mathrm{pi}$ & ANis \\
\hline $28 \mathrm{G} 1 \mathrm{~A}$ & Poten:enten & 225 & U. & $.22 .03 P C$ & AMPS \\
\hline $2861 \mathrm{~A}$ & Potaningon & $22+1$ & U & $2.10 \mathrm{AC}$ & ANCS \\
\hline ZEG 1A & Promannam 14 & हथी & $\mathbf{u}$ & $+1.4 \mathrm{PCL}$ & ANCS \\
\hline 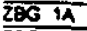 & Promolicinim is & हर्थ & Ui & $1.35 \mathrm{PCC}$ & ANES \\
\hline $200 \mathrm{~A}$ & Promentum-140 & $2 \sqrt{200}$ & Un & $-2.0 \mathrm{AC}$ & ANDS \\
\hline $2 B G 1 A$ & Promeristem-140 & 2he & Ü & $4.50 / P C$ & ANNDS \\
\hline 28614 & 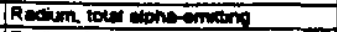 & $22 \Omega$ & & $55.51 / P C$ & ANES \\
\hline ZBG & Rechm-220 & चय & & $7.799 \mathrm{Ca}$ & ANDS \\
\hline $2 B G 9$ & Recingm-228 & $2 / 20$ & & $8.09 P C$ & AÑBS \\
\hline BEG & Rutheniminios & $2 / 2 \times$ & $\mathbf{u}$ & $-1.201 P C 2$ & $\operatorname{Ann} 3$ \\
\hline ZQG 1A & Ruthenim-10s & 2220 & Uं & 12.05 PCL & ANDS \\
\hline ZEG $1 \mathrm{~A}$ & sodingen & 2200 & $\underline{H}$ & $-1,496$ & AN:S \\
\hline ZEG $1 \mathrm{~A}$ & Sodin-22 & $2 \sqrt{2}=$ & U్ & $0.39 P C$ & ANES \\
\hline ZEG 1A & Tecomenumes & 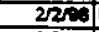 & UI & 10.7]PC & ANDS \\
\hline ZEG $1 A$ & 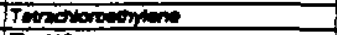 & $22 \pi 0$ & $\mathbf{u}$ & sual & ANS \\
\hline ZES IA & Thin 113 & 220 & $\mathbf{u}$ & $4.5 \mathrm{PC}$ & ANS \\
\hline 28014 & $\operatorname{Tin} 113$ & 2 and & $\mathbf{u}$ & $4.4 \sqrt{C E}$ & ANCS \\
\hline $2001 \mathrm{~A}$ & Tolom & $2 \pi 20$ & $\bar{u}$ & SIUE & ANis \\
\hline 204 & 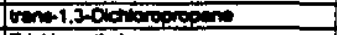 & 2us & $\bar{u}$ & S|ज्व & ANS \\
\hline 20014 & 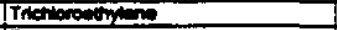 & $2 \sqrt{200}$ & $\mathbf{u}$ & SUa & ANS \\
\hline 280 iA & 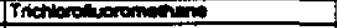 & 220 & $\mathbf{U}$ & STिa & ANSS \\
\hline $2901 \mathrm{~A}$ & $x$ & $22 \times$ & U & Stuce & AN: \\
\hline 28614 & Youmes & 2200 & U: & \begin{tabular}{l|l}
-1.02 & $F C$ \\
\end{tabular} & ANA \\
\hline 20014 & 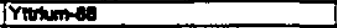 & $2 \pi 0$ & $\overline{\mathbf{u}}$ & T.4|PC & Ancs \\
\hline $296 \sqrt{4}$ & Znoes & $22 \pi$ & Us & $-2.14 P C$ & ANS \\
\hline 28014 & Znness & 2य) & ज & $1.75 \rho C$ & ANA \\
\hline $2061 \mathrm{~A}$ & zaconimines & $2 \sqrt{2} \approx 0$ & जि & $.2 .52 / \mathrm{PC}$ & ANS \\
\hline 28014 & Zecontions & 2द्यु & US & $5.3 \times 1 \mathrm{CL}$ & $\sqrt{A N B}$ \\
\hline 2002 & 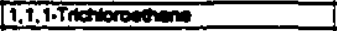 & $2 / 20$ & U & 5Uि & ANS \\
\hline 2832 & 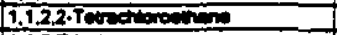 & $2 \pi^{\circ}$ & U. & 5 Ua & AN: \\
\hline 2062 & 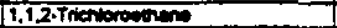 & $2 \pi 20$ & $\mathbf{U}$ & डणिद & ANS \\
\hline ZEG 2 & 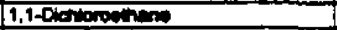 & $2 \sqrt{20}$ & $\mathbf{u}$ & SU्द & NAS \\
\hline 2002 & 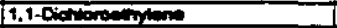 & $2 \mathrm{sen}$ & u & suti & AN: \\
\hline 2002 & 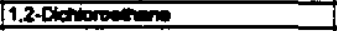 & $2 \sqrt{2}$ & $\mathbf{U}$ & stuat & $A=3$ \\
\hline 2932 & 1,2-Detroventionse & 2ह1 & $\mathbf{u}$ & SUि & AN: \\
\hline 2962 & 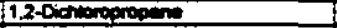 & 2थ्य & $\mathbf{U}$ & Sप्ug & ANES \\
\hline 2902 & 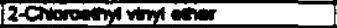 & 2यां & $\mathbf{U}$ & 100 & ANis \\
\hline 2002 & Actimen-232 & 2220 & $\mathbf{U}$ & $-1.04 \sqrt{C a}$ & AN. \\
\hline 2002 & 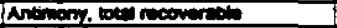 & $2 \sqrt{2}=1$ & $\mathbf{U}$ & 27!va & ANS \\
\hline 2002 & Animinimine 124 & $2 \sqrt{2}$ & कि & $.5 .6 \mathrm{~d} F \mathrm{C}$ & AN:S \\
\hline 2002 & Antinos 125 & 2त्2: & $\mathbf{H}$ & $.2 .619 C$ & aris \\
\hline 2062 & 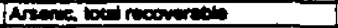 & 2का & $\mathbf{U}$ & Wive & ANDS \\
\hline 2802 & 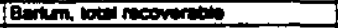 & $2 \sqrt{2}$ & & Q.1UEL & anes \\
\hline 2902 & Betum 133 & $22 \%$ & $\mathbf{u}$ & 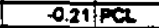 & ANOS \\
\hline 282 & Beraing & 2थ: & U & SUE & AN: \\
\hline 2002 & 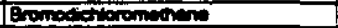 & एक्ता & Iu & Sज् & ANes \\
\hline 2002 & Bromotom & $2 \sqrt{2}$ & U & S.UCE & ANaS \\
\hline 2862 & 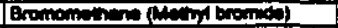 & 2त्या & I0 & totue & ANB \\
\hline 2EG 2 & 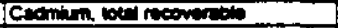 & $2 \sqrt{250}$ & (0) & $4.7 \sqrt{0}$ & ANS \\
\hline 2862 & Certon torectionde & $2 / 200$ & U & डाण्a & ANS \\
\hline 2002 & Contemi4 & $22 \times 3$ & (1) & $0.7 \overline{A C}$ & tes \\
\hline$\overline{2 E G 2}$ & Cesin 134 & 2/2:3 & U & $0.03 \mathrm{PCL}$ & ANG \\
\hline $2 E Q_{2}$ & Casin 137 & $2 \sqrt{200}$ & $\mathbf{u}$ & $2.42 / \overline{C L}$ & NT:S \\
\hline$\angle E G 2$ & 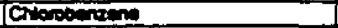 & $2 \pi \sqrt{2}$ & $\bar{u}$ & $5 \longdiv { C _ { 2 } }$ & ANS \\
\hline 2062 & Crapenten & 2यi & U & 10ण्द & $A \times S$ \\
\hline 2002 & 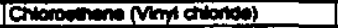 & 2ह: & $\bar{U}$ & 10,Ua & ANOS \\
\hline 2002 & Cribententin & 2है: & $\bar{\omega}$ & 5ण्2 & Nins \\
\hline 2602 & 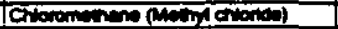 & $2 \pi$ i & U & TOUE & NWE \\
\hline 2062 & 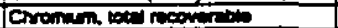 & $2 \sqrt{20}$ & J & 1.5) & ANG \\
\hline 2092 & 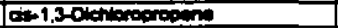 & $2 \sqrt{2 i n}$ & $\overline{0}$ & 5ivat & ANS \\
\hline 2902 & $\cos -57$ & $2 \Omega$ 2तi & $\mathbf{U}$ & -1.00102 & ANB \\
\hline 2062 & $\cos 25$ & $22 \pi$ & $\mathbf{U}$ & $1.017 C$ & NAS \\
\hline 2062 & $\cos 160$ & 2था & $\mathbf{U}$ & $1.4 P F C$ & AN: \\
\hline 2052 & 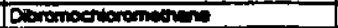 & 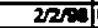 & $\mathbf{U}$ & Stua & An: \\
\hline 2062 & 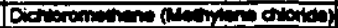 & 225 & $\tilde{u}$ & 20.7 पa & N:S \\
\hline 2002 & Eingontions & $22+2$ & $\bar{U}$ & SUa & ANO \\
\hline 2002 & Eurcoitustis2 & $2 / 2: 3$ & $\overline{\mathbf{u}}$ & $11.29 A$ & ANS \\
\hline $2 E 02$ & Europinis & $2 / 2: 0$ & $\mathbf{U}$ & $-1.53, P C$ & A:S \\
\hline $2 \mathrm{EO} 2$ & Europinmis & 2ह5: & $\mathbf{u}$ & $0.51 P C$ & ANBS \\
\hline 2002 & Grosis an & 2था & $\mathbf{w}$ & $0.70 / \overline{C L}$ & $\operatorname{An} S$ \\
\hline 2002 & 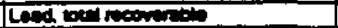 & $2 \Omega$ & $\bar{u}$ & 47 ua & NQS \\
\hline 2062 & Loed212 & $2 \sqrt{2}$ & $\mathbf{U}$ & $1.20 \mathrm{PC}$ & ANS \\
\hline 2002 & Marpinatest & $2 \Omega i$ & $\mathbf{U}$ & $0.04 / \mathrm{DC}$ & ANE \\
\hline ZEG 2 & 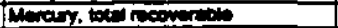 & $22 \pi$ & U & $0.90 \mathrm{UL}$ & ANES \\
\hline 2062 & Neptritim 230 & 2था & $\bar{\omega}$ & $.2150 \mid \not C$ & AN:S \\
\hline 2062 & 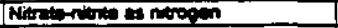 & $2 / 20$ & & 79:Ua & AN:S \\
\hline 2962 & Nitries ete nowogen & $2 \sqrt{2}$ & $\bar{u}$ & ZणUिa & ANQS \\
\hline 2062 & Nonnoledis ben & 2थ्ता & Uiv & $1.301 \mathrm{PC}$ & IANOS \\
\hline $28 \mathrm{EG}$ & pit & $2 \sqrt{2}=$ & I5 & $5.291 \mathrm{FH}$ & ANDS \\
\hline $2 B Z_{2}$ & 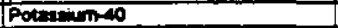 & $22=0$ & iu & 18.4APC & ANDS \\
\hline 2802 & Promminam 14a & $2 \sqrt{251}$ & Uि & $2.591 \mathrm{AC}$ & Anis \\
\hline 2902 & Promeniem 140 & 220 & us & $-3.91 \%$ & Anes \\
\hline$\angle B G 2$ & 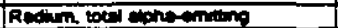 & $2 \sqrt{2}$ & $\bar{u}$ & $4.37 \sqrt[P C]{ }$ & ANOS \\
\hline 2EG 2 & 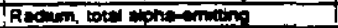 & $2 \sqrt{2}=0$ & U & $4.72 \mid P C Z$ & ANBS \\
\hline
\end{tabular}

- WSRC-RP-99-00002 


\begin{tabular}{|c|c|c|c|c|c|}
\hline WELL & ANirire & OATE & OUAUFIER & HRESULT IUNII & FORMAT \\
\hline $2 B G_{2}$ & Redimm-220 & 220 & & $0.7 \mathrm{FAC}$ & AN:S \\
\hline 2062 & $1 \operatorname{Rocium-220}$ & $2 \sqrt{200}$ & & $1.12+P C$ & ANS \\
\hline 26 S2 $^{2}$ & Racimm-228 & $2 \pi 00$ & UT & $.123 P C$ & ANOS \\
\hline $2 B O 2$ & Recim-222 & $22 \times 0$ & $\mathbf{u}$ & 1.10170 & An:S \\
\hline ZEG 2 & Ristoment 106 & $22 \pi$ & $\mathbf{U}$ & $5.6 \sqrt{\mathrm{C} C}$ & ANOS \\
\hline 2062 & 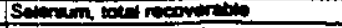 & $2 / 2 \times 3$ & 0 & OUिद्र & ANes \\
\hline$\angle B G 2$ & 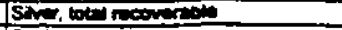 & 2250 & IJ & 1.7) & AMS \\
\hline ZEG 2 & Soxim-22 & 225 & U & 0.57 ल्ल1 & ANCS \\
\hline ZEG 2 & 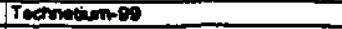 & 2280 & $\mathbf{U}$ & $0.30 \mathrm{PC}$ & Anes \\
\hline ZEG 2 & Tectinim-s & $22 \pi$ & $\bar{y}$ & OPCL & ANis \\
\hline $2 B$ C & 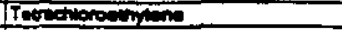 & $2 \sqrt{201}$ & IU & 5ाप्a & ANS \\
\hline 2862 & Tn:113 & $22=0$ & U & $-0.0 \not C 1$ & ANES \\
\hline ZEG 2 & Tolane & $2 \sqrt{2}$ & $\mathbf{5}$ & 5 जut & ANis \\
\hline \#6 2 & 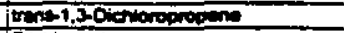 & $2 \sqrt{2 \pi}$ & U & 5) & $A N_{1}=$ \\
\hline ZEG 2 & 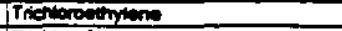 & $2 \sqrt{20}$ & U & s)e & ANS \\
\hline 2862 & 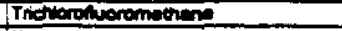 & $2 \sqrt{2}=0$ & $\bar{u}$ & Stफ्C & ANES \\
\hline 2002 & $x_{y}$ & $2 \sqrt{25}$ & U & SUिन & ANS \\
\hline ZEG 2 & Yoinen & $22 \pi$ & TI: & $0.02 P C$ & Ants: \\
\hline ZEG 2 & inges & $2 \sqrt{20}$ & a & $0.51 P C$ & ANDS \\
\hline 2002 & Zinoonamis & 2200 & (1) & -2.74 FCL & Anis \\
\hline 2001 & 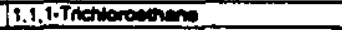 & ariel & I0 & 5 Ua & ANAS \\
\hline 2001 & 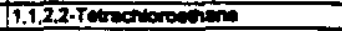 & 44in & iv & Sप्क & ANES \\
\hline 2801 & 1.1,2-7richiouterese & 840 & $\bar{U}$ & Siver & AN? \\
\hline$\overline{Z E O} 1$ & 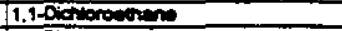 & yase & 0 & अचू & ANCB \\
\hline $2 E O_{1}$ & 1,1-Obtionowions & Vars & v & 5 Ua & Ans \\
\hline 2001 & 1.2- & swas & II & $5 \longdiv { U E }$ & ANDS \\
\hline 2001 & 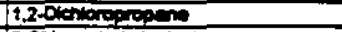 & yisn & $\bar{u}$ & 5uan & ANS \\
\hline 2961 & 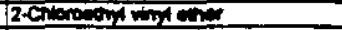 & $\operatorname{lach}$ & U & TOU Ua & Anis \\
\hline 2061 & Actinin-220 & Man & IU: & $-4.45 \mid P C$ & ANos \\
\hline 201 & 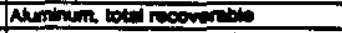 & 84 & J & $22.210 \mathrm{G}$ & ANe \\
\hline 2001 & 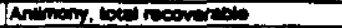 & Noid & $\bar{v}$ & 27/U日: & An:s \\
\hline 2801 & Antinow 124 & rea & U & $3.30 \mathrm{AC}$ & ANis \\
\hline 2801 & Axison-125 & QWais & ư: & $.2 .01 P C$ & ANGS \\
\hline 2001 & 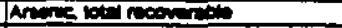 & 14:Dil & I0 & 40 & ANis \\
\hline 2801 & 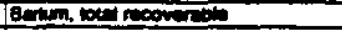 & 84asis & & 9.7Uिa & 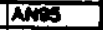 \\
\hline $296:$ & Qontein-133 & Wore & $\mathbf{U}$ & $2.41 P C$ & NAS \\
\hline 2801 & Baritine & 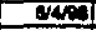 & Uu & SUG & Anis \\
\hline EQC 1 & Fomedration & Woil & 0 & SUCL & ANG \\
\hline 201 & Eronotim & owin & $\mathbf{U}$ & 5ुUa & AND \\
\hline 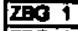 & 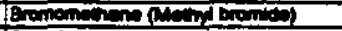 & twing & 0 & 100 & AN:S \\
\hline ZEO 1 & 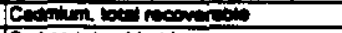 & 2yid & 0 & 4.7 UCL & ANTS \\
\hline 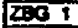 & 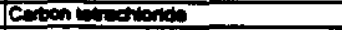 & क्w & $\bar{U}$ & 5ua & ANos \\
\hline 2801 & Continim & wain & $\mathbf{U}$ & $1.90 P Q$ & AC:D \\
\hline 2001 & Contan-134 & gria & $\mathbf{w}$ & $2.17)$ & ANis \\
\hline 2031 & $C_{0}=137$ & 94a & Wu & $2.02 \sqrt{2}$ & AN \\
\hline 2008 & 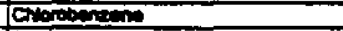 & ren: & (U) & 5ाua & ANT \\
\hline 2801 & 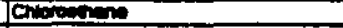 & 8wi: & $\mathbf{U}$ & 10UE & AN. \\
\hline 2001 & 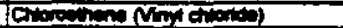 & gwi & 0 & $1 0 \longdiv { a }$ & $\sqrt{A \times 3}$ \\
\hline 2001 & Chiorolom & vail & $u$ & S|ण्: & ANS \\
\hline 2.01 & 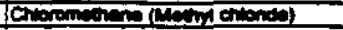 & सब. & $\mathbf{U}$ & 10lua: & AN. \\
\hline$\angle \mathrm{EO} 1$ & 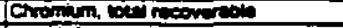 & Eavi & $\mathbf{U}$ & 7 Ua & ANis \\
\hline 2861 & 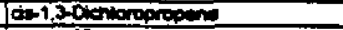 & aris & U & 5 प्र: & IANS \\
\hline ZEO 1 & $\cos 257$ & Whel & $\mathbf{G}$ & 1.8IFC & Ans \\
\hline 2861 & $\cos \sin ^{2}$ & Qus & UT & $0.7 \overline{F a}$ & ANOS \\
\hline ZEG 1 & $\cos 10$ & QWin & $\mathbf{U}$ & $2.32 \mathrm{PC}$ & ANCS \\
\hline $2 \mathrm{BO} 1$ & 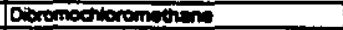 & Bures & $\mathbf{U}$ & S) UE & ANis \\
\hline 2001 & 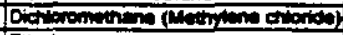 & raina & $\underline{u}$ & 3.87 LG & ANOB \\
\hline 20051 & Expentang & Man & $\mathbf{u}$ & 5 प्र & ANS \\
\hline 2001 & Exropin 152 & Q⿻is & $\mathbf{U}$ & $.10 .2 P C$ & ANS \\
\hline 2001 & Extinm-134 & Q4in & $\mathbf{Q}$ & $.1 .15 P C$ & ANos \\
\hline ESG 1 & Europinm-150 & क्राiा & U & $6.3 P \mathrm{C}$ & An:S \\
\hline 2861 & Grous Exe & Fwin & & $1.05 \mathrm{PC}$ & Anis \\
\hline ZEG 1 & 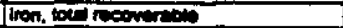 & Qwiva & & बाप्स & ANB \\
\hline ZEO 9 & 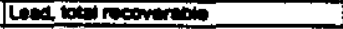 & Whis & & $9.30 \mathrm{C}$ & ANDS \\
\hline 206 1 & Lood 312 & QW:DI & $\mathbf{U}$ & $.1 .05 \mid F C$ & ANES \\
\hline EQG 1 & Henchens & 840 & $\mathbf{U}$ & $0.70 \mid F C$ & ANS \\
\hline 2869 & 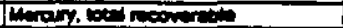 & Qual & U, & $0.7 \mathrm{Ua}$ & AN: \\
\hline 2001 & Nequin-24 & कक्षा & $\mathbf{\omega}$ & $7.53 \mathrm{FCL}$ & A.: \\
\hline 2001 & 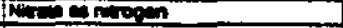 & BND & $J$ & 1440 US & NNS \\
\hline 2801 & 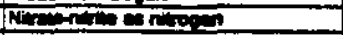 & Wriv & $J$ & 1300 Ua & ANTS \\
\hline ZEG 1 & 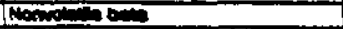 & and & & A,APCE & $\sqrt{A+B S}$ \\
\hline $2 \mathrm{EO} 1$ & Dh & mol & 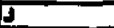 & $5.7 / \mathrm{FH}$ & AN: \\
\hline $2 \mathrm{BO}$ & Potem no & QWe & $\mathbf{U}$ & .27 .400 & AN \\
\hline 2001 & Proming & Thin & $\boldsymbol{T}$ & $0.32 / \overline{C a}$ & AN: \\
\hline 2861 & PFon Fine & and & (w) & $-0.84 \mathrm{PC}$ & ANis \\
\hline $266_{1}$ & Ratim & aris & $\overline{\mathbf{w}}$ & $0.521 \mathrm{PCL}$ & $\overline{A N}$ \\
\hline 2061 & 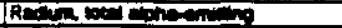 & क्ष & & 1.0Aम्C & ANB \\
\hline$\angle B O \quad 1$ & Res $=20$ & gen & $\mathbf{U}$ & $4.30 \mathrm{~Pa}$ & AR:S \\
\hline 2061 & Ruliminio & erea & Q & $7.1 / \mathrm{AC}$ & ANCS \\
\hline 2001 & 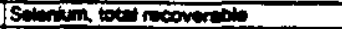 & vail & $\overline{0}$ & Q & AN \\
\hline 2801 & 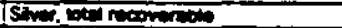 & Y40: & $\mathbf{u}$ & 5.uch & $\sqrt{A-3}$ \\
\hline 2061 & Solvinge & $\Delta \omega$ & $\overline{\mathbf{u}}$ & $-0.40 \mid P C$ & Ans \\
\hline 2001 & Techloumea & vain & Ü & $1.32 \mid P C L$ & ANDS \\
\hline ZES 1 & ITrectoroming & grat & $\bar{U}$ & 5:Ua & ANDS \\
\hline 2801 & $\operatorname{Tn} 113$ & Mand & UT & $-0.61 P C L$ & ANis \\
\hline 2801 & Tolven & Man & $\mathbf{u}$ & 5ीUG & ANoS \\
\hline 2EG 1 & 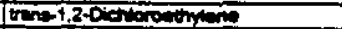 & NaDe & $\mathbf{U}$ & 5) UC: & $\sqrt{\text { ANBS }}$ \\
\hline EQG 1 & 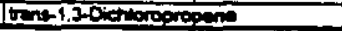 & OWand & & 5)पद्र & ANOS \\
\hline
\end{tabular}

WSRC-RP-99-00002 


\begin{tabular}{|c|c|c|c|c|c|c|}
\hline WELI & TANALYTE & OATE & QUAUFIER & IRESULT & UNHT & TFORMAT \\
\hline 2861 & Trichiorow mpene & vanto & iv & & siva & ANOS \\
\hline ZBG 1 & Ineriopotiverometren & $\sin 20$ & IU & & 5.UGL & ANSS \\
\hline 2861 & ixyment & $\sqrt{4 x}$ & IU & & $5 ! U G$ & IAMDS \\
\hline 200 & Yrovmons & vero & IUt & -3.00 & DIPCL & ANOS \\
\hline 2861 & Eneses & $\sin$ & U & -0.70 & DPCA & ANAS \\
\hline $20 G 1$ & Zrounimas & ativ & U & 0.23 & $3 \mathrm{PCL}$ & AN \\
\hline 2062 & 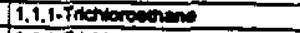 & ह10 & IU & & IIUAL & ANDS \\
\hline 2 EG 2 & 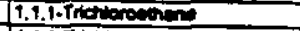 & 2000 & Iu & & डण्ब & ANS \\
\hline 20,2 & 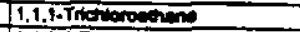 & vavis & W & & S:UG & ANSS \\
\hline 2062 & 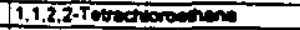 & Wwiv & U & & 1) & ANOS \\
\hline 2062 & 1.1.2.2-Ternachionom & gave & To & & 5.vat & ANS5 \\
\hline 2062 & 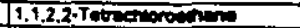 & $8 \times$ & tw & & Sपिक् & ANSS \\
\hline 2862 & 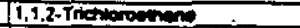 & 20 & iv & & 1यद & ANDS \\
\hline ZEG 2 & 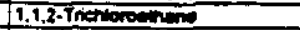 & ane & I0 & & Sाण्द & AMS \\
\hline$\overline{2062}$ & 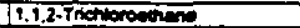 & (2/4) & कि & & 5णिa & ANSS \\
\hline ZEG & 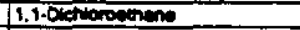 & 840 & $\bar{U}$ & & 1uet & ANDS \\
\hline $2 \overline{802}$ & 1,8-0ichoromenten & श्रफ & tiv & & sluat & ANDS \\
\hline 2602 & 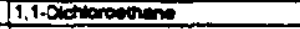 & 20 & tw & & s]ua & $\sqrt{\text { ANGS }}$ \\
\hline 2802 & 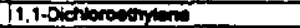 & Q45 & I0 & & 1) & ANAS \\
\hline 2862 & 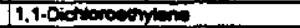 & $\sqrt[4]{4 \sqrt{x}}$ & $\bar{u}$ & & Sप्र & anis \\
\hline$\overline{2062}$ & 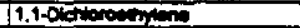 & 20 & w & & 5 Uत्र & ANDS \\
\hline 2002 & 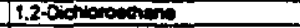 & $2 \sqrt{\infty}$ & IU & & IUत्र & ANOS \\
\hline 2002 & 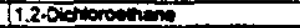 & $\operatorname{lon}$ & iv & & Sप्रद & ANOS \\
\hline $2 E 62$ & 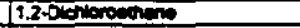 & aso & IW & & 5ीणCL & $\widehat{A N S}$ \\
\hline$\overline{\angle E C 2}$ & $1,2-01010100000$ & प्रक्त & $\bar{U}$ & & Tuat & ANAS \\
\hline 2862 & 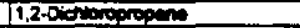 & Wwis & u & & Sण्C & TANOS \\
\hline 2002 & 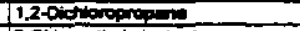 & Twe & w & & 5ॉण & ANDS \\
\hline$\overline{282}$ & $2-C^{2}=0$ & Q6in & I0 & & अण्द & ANo \\
\hline$\angle B C_{2}$ & 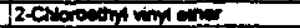 & 90 & U & 10 & OUC2 & ANDS \\
\hline$\angle E 62$ & 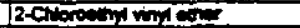 & IकW & W & 10 & ofuct & ANCS \\
\hline सQ2 & Actinitin-22: & Eक्w & Ū & -0.47 & निक् & TANBS \\
\hline ZEG 2 & Achinge20 & प्रक्ष & प1 & 1.20 & S]FC & Anis \\
\hline 2012 & Acentinges & VWn & U & 11.10 & Ifन & ans \\
\hline 2002 & Acinginzan & Q4in & U & 24.4 & AFCL & AN \\
\hline 2032 & 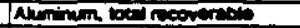 & gen & J & 12.1 & TUGL & AX:S \\
\hline \$192 & 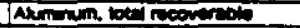 & $v_{40}$ & J & 16.8 & बण्द & ANS \\
\hline 2862 & 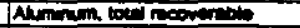 & $\operatorname{los}$ & 12 & 4.7 & $7 \mathrm{Ual}$ & Anes \\
\hline 202 & 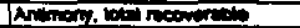 & o. & 0 & 0.2 & 30 & ANis \\
\hline 2002 & 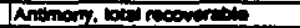 & प्रin & U & 27 & 7ण्ड़ & ANis \\
\hline$\overline{2002}$ & Arinerin 129 & $\operatorname{lin} 2$ & $\bar{U}$ & .2 .04 & AFCL & AN:3 \\
\hline$\overline{Z B 2} 2$ & Aximation 120 & 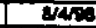 & U & 0.94 & FC & ANS \\
\hline 202 & Ansooser 126 & बin & IO & 3.57 & $7 \bar{C}$ & Awis \\
\hline 202 & Aninevili25 & arin & נU & -2.80 & APCL & AW: \\
\hline 2002 & Antroint125 & $\operatorname{lin}$ & $\mathbf{u}$ & -1.82 & APC & ANPS \\
\hline 2062 & Aningir 125 & 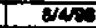 & $\tilde{u}$ & -1.02 & 2क्C & ANis \\
\hline$\overline{2 B G 2}$ & Axtinery 125 & $\sqrt{4: 2}$ & 10 & 1.8 & DPC & AN: \\
\hline 2002 & 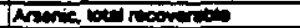 & Wwing & iv & 3 & 3ण्द & ANS \\
\hline 2862 & 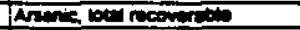 & Vain & 10 & $\$ 0$ & कण्दL & ANis \\
\hline 2062 & 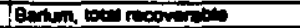 & $\log$ & & 5.9 & TUE & ANDS \\
\hline Z62 & 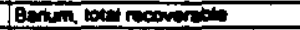 & Evin & & 6.4 & A) & $\tan 3$ \\
\hline 2062 & 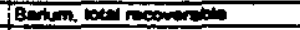 & Vas & & 5.34 & IU⿺𠃊) & Anes \\
\hline 2802 & gexpm-133 & wain & it & -6.42 & 2 PC & ANAS \\
\hline 2002 & Tentsm 13 & INA & गि & -4.30 & $\overline{B C}$ & ANSS \\
\hline 2602 & Benming 133 & हैin & $\bar{T}$ & -0.50 & P्C & ANBS \\
\hline 2862 & Baresen & $\sqrt{425}$ & U & & iUज & ANDS \\
\hline$\overline{802} 2$ & Berrens & ow: & U & & 5ive & Ales \\
\hline ZEG 2 & Benowe & $\sqrt{4 \times 20}$ & Iu & & 5ua & ANis \\
\hline 2802 & Bronodicteromethe & aDis & IU & & 1णिa & TANS \\
\hline 2602 & 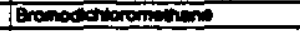 & Wait & $\overline{0}$ & & ș्a & ANeS \\
\hline 2002 & 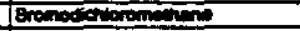 & vain & w & & $5 \longdiv { \overline { L } }$ & ANes \\
\hline 2062 & Bromeitem & 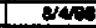 & u & & ITE & ANES \\
\hline 2862 & Eoconotion & $\operatorname{les}$ & tu & & suल & ANis \\
\hline ZEG & Bromoivin & Q⿻ & W & & 5 tua & ANis. \\
\hline 2182 & 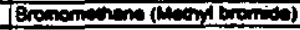 & We & 0 & & 1 Ua & An: \\
\hline 2802 & 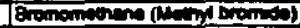 & QTin & ]U & 10 & ox & ANS \\
\hline 2802 & 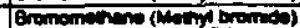 & vat & $\boldsymbol{\omega}$ & 10 & ove & AN:S \\
\hline 2802 & 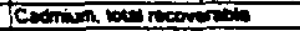 & exis & 可 & & उण्द & Nis \\
\hline 2802 & 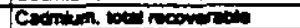 & 14 & $\sqrt{0}$ & 4.7 & 7ण्य & ANis \\
\hline 2002 & 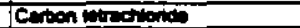 & Toin & U & & 1]Uद & ANis: \\
\hline 2862 & 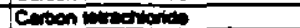 & wis & $\bar{U}$ & & Sप्रा & AN:3 \\
\hline 2062 & Comen & 60 & III & & 5ue & An: \\
\hline $200 \overline{2}$ & Contenim & 80 & In & .3 .00 & उब्र & Anis \\
\hline 2002 & Conmin & 20 & U & 2.63 & 3 PCL & ANOS \\
\hline 2802 & Connen's & प्रत & in & 7.47 & $7 \overline{P C}$ & ANS \\
\hline$\angle E G 2$ & Conoth 14 & 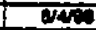 & in & 7.5 & $5 \mid P C$ & ANS \\
\hline 2862 & Cenanion 134 & vas & U & -1.20 & $D F C$ & ANis \\
\hline 2062 & $C_{0}{ }_{113} 134$ & gais & 30 & 0.22 & ZPQ & daris \\
\hline 2802 & Cotemish & प्रत: & Un & 3.4 & $A P C$ & ANis \\
\hline 2032 & Coming 13 & QWin & נtw & 0.002 & DPC & AMBS \\
\hline 2062 & Cation 137 & WQ: & $\omega$ & 0.397 & 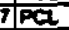 & ANis \\
\hline 2002 & Conson 137 & 20100 & U & 1.03 & $\sqrt{P C}$ & ANS \\
\hline $28 S_{2}$ & Coemm 137 & एक्जि & $\bar{x}$ & 2.15 & S)PCI & ANS \\
\hline 2962 & Cogam-137 & Nain & U & 2.97 & $7 \overline{C L}$ & ANos \\
\hline 2002 & 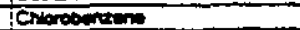 & 2003 & tu & & 1UE & ANAS \\
\hline 2062 & Conoropintern & 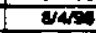 & 10 & & SUCt & ANAS \\
\hline 2862 & 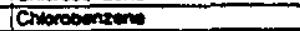 & बक & ww & & 5ीuat & ANGS \\
\hline 2002 & Chioronen & 0 & IV & & TUEL & ANBS \\
\hline$\angle B G 3$ & chionosines & $34 \sqrt{20}$ & tu & & OUिद & TANS \\
\hline
\end{tabular}

WSRC-RP-99-00002 


\begin{tabular}{|c|c|c|c|c|c|}
\hline WELL & IANALYE & QATE & OUAUFIER & iRESULT :UNIT & Format \\
\hline 2102 & Chorowentere & Quase & IUS & 10iug & ANOS \\
\hline$\overline{2002}$ & Chioromene nemi atondal & 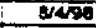 & Ju & 10G & ANDS \\
\hline 2862 & 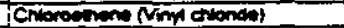 & प्र490 & iv & णियद & ANOS \\
\hline 2062 & 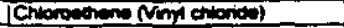 & 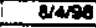 & ju & 10ंUGL & ANDS \\
\hline ZOG 2 & Crivorotoin & Non & iv & TUEL & ANDS: \\
\hline ZBG 2 & Cntoroton & ब्रक्य & ]v & डUिद्य & ANES \\
\hline$\overline{E B S}$ & Cropion & 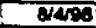 & נய & 5)णदh & ANDS \\
\hline$\overline{Z E Q 2}$ & 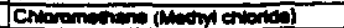 & VWas & vo & 1 U⿺ & ANes \\
\hline 202 & 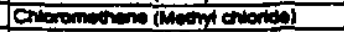 & arsis & 0 & 10,UGh & ANeS \\
\hline$\overline{2 E O 2}$ & 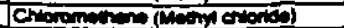 & Wक्ष & w & TOULL & AN:S \\
\hline 2862 & Chroming neter nooverate & 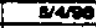 & dJ & 10 प्a & ANos \\
\hline $2 B G 2$ & 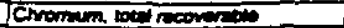 & $\operatorname{yar}$ & U & 1.71UGL & IANOS \\
\hline $2 B G 2$ & 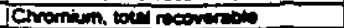 & Wwis & 0 & 7|va & ANoS \\
\hline 2BG2 & 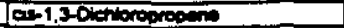 & vai: & $\sqrt{\mathbf{u}}$ & TUa & ANS \\
\hline$\overline{2 B G 2}$ & 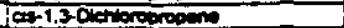 & $y=3$ & to & SUGL & ANos \\
\hline 2062 & cis 1.3-Oichtoropopemen & QW & w & S|णि & ANES \\
\hline ZEG 2 & $\cos =57$ & 84: & $\mathbf{\omega}$ & 0.10 FC & ANES \\
\hline 2862 & Cobents & 200 & $\sqrt{u}$ & $0.001 P C$ & ANOS \\
\hline 2862 & $\cos 557$ & Qwave & U & $0.755 \mid P C$ & ANDS \\
\hline 2012 & Cosens & Vare & U & 1.52 PCL & ANes \\
\hline 2002 & $\cos \cos ^{2}$ & 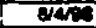 & $\bar{U}$ & $0.02 \not C$ & ANDS \\
\hline 2002 & $\cos 25$ & $\mathrm{BW}$ & U & $1.02 / 9$ & $A 0$ \\
\hline 2862 & $\operatorname{cosen} 5$ & 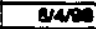 & $\mathbf{u}$ & 1.53/PCI & ANis \\
\hline 4002 & Cabesen & visia & U & -2.SPFC & ANS \\
\hline 2802 & cobensen & $\sqrt{a}$ & tit & $0.33 P C$ & ANG \\
\hline 2062 & $\cos \theta 0$ & प्रत: & ITU & $8.2)$ PC: & ANOS \\
\hline ZEG 2 & $\cos \theta 6$ & ग्रा & iti & 1.71 PC & Aw:S \\
\hline$\overline{Z E B 2}$ & Doromedionen & Wwa & ivo & TUa & ANOS \\
\hline ZEO 2 & Doromectionom & g/4:2 & iv & stua & Ans \\
\hline$\overline{E Q O Z}$ & 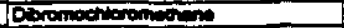 & ase & W & Sाü & ANCS \\
\hline$\angle E 62$ & 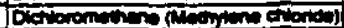 & Whe & 0 & 4.02Uू & TANS \\
\hline ZEG & 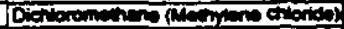 & 20 & U & $1 \mathrm{UE}$ & ANS \\
\hline 2862 & 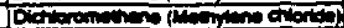 & 90 & w & 3.94Uद्स & TWS \\
\hline 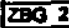 & Etringen & 00 & $\overline{0}$ & 1Uat & 1403 \\
\hline ZES 2 & 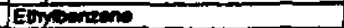 & 840 & 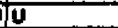 & 3)्al & ANGS \\
\hline$\overline{\mathrm{ZEO} 2}$ & Etrivention & प्रक & $\omega$ & SUat & Ans \\
\hline 2002 & Erroginm 152 & एकin & U & $.17 .53 \hat{\alpha C}$ & ANes \\
\hline 2802 & Exropin-152 & Swatio & U & $.2 .77 P C$ & ANes \\
\hline ZEO 2 & Erotion-152 & Wकi: & IUT & $3.10 \mathrm{PC}$ & Anes \\
\hline 203 & Expoinisis2 & Bris? & U & $12.31 \mathrm{AC}$ & ANS \\
\hline 282 & Exogenimis & 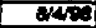 & $\bar{U}$ & $5.521 F C$ & ANOS \\
\hline $200 \overline{2}$ & Evodisis & एक्ज & U & $-1.4 \mathrm{FC}$ & AM: \\
\hline 2002 & Erichents & garia & U & 3.02 PC & Th: \\
\hline 2802 & 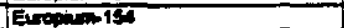 & $\operatorname{vas}$ & W & $6.03 P C$ & AND \\
\hline$\overline{2032}$ & Expondis & क्रक्त & U & $.152, \mathrm{PC}$ & ANAS \\
\hline 2802 & Evo.enmis & Waris & Ui & $1 . 2 1 \longdiv { P C }$ & 1003 \\
\hline 2052 & Evroginn 15 & Vwin & W & $2.07 / \overline{P C L}$ & ANiB \\
\hline 2002 & Errogin-15s & Wars & $\mathbf{U}$ & 2.9PE & ANOS \\
\hline $20 B 2$ & Grosen & क्रक्ड & in & $0 . 3 2 \longdiv { 2 }$ & ANOS \\
\hline$\overline{E 0} 2$ & Groen & P4in & U & $0.525 \overline{C a}$ & ANS \\
\hline 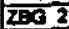 & Groes & owan & & $0.775 \mid F C$ & AN: \\
\hline$\angle E O 2$ & Groes on & owia & & $0.03 \sqrt[F C]{ }$ & ANB \\
\hline ZES 2 & Gool an & vain & & $1.22 \sqrt{\mathrm{PCL}}$ & AN \\
\hline$\overline{2102}$ & 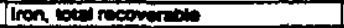 & yans & 5 & 24.9002 & AN \\
\hline 2002 & 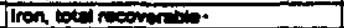 & Uase & J & Q4.310 & AN:B \\
\hline 2862 & iron, totel recover bis & yan & & 33.81uci & ANBS \\
\hline 2062 & Leed, wot recoverétio & owas & IJ & 0.877 Ua & AN:B \\
\hline 2802 & 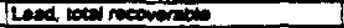 & gaves & W & MIVA & AMS \\
\hline $28 C^{2}$ & Loed-212 & Q14:0 & IU & $.1 .91 / a$ & ANB \\
\hline $20 \mathrm{ES}$ & Lent212 & पas & IU & $5.41 / \overline{P C}$ & ANes \\
\hline ZES 2 & Loed-212 & exin & II & $7 . 6 7 \longdiv { P C A }$ & AN:S \\
\hline ZEG 2 & Leeci212 & MWD & & $10 . \infty \mathrm{FC}$ & ANis \\
\hline ZEG 2 & Meromens & ven & TU & $-2.80 \mathrm{PC}$ & ANS \\
\hline$\overline{Z 192}$ & Moriming & Was & $\boldsymbol{\omega}$ & $-1.20 / P C$ & $\operatorname{AxS}$ \\
\hline 2062 & Mentenest & हand & $\omega$ & \begin{tabular}{l|l}
0.72 & $\mathrm{PC}$ \\
\end{tabular} & ANi:S \\
\hline 2902 & 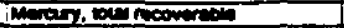 & Wan & $\bar{U}$ & $0.7 \mathrm{Ua}$ & An:s \\
\hline 2802 & 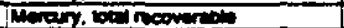 & Pan: & & 0.7 Ua & Anis \\
\hline 2862 & Nepentin 230 & क्w & $\mathbf{W}$ & $\$ 3.61 P a$ & ANOS \\
\hline 2802 & Nequinzas & $8 \times$ & $\mathbf{U}$ & .12010 & AN:S \\
\hline 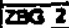 & 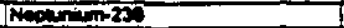 & ewa & U & $-1.07 \mathrm{FC}$ & AN:5 \\
\hline$\overline{Z E O} 2$ & Nerenoren & van & 1 & 570) & AN: \\
\hline ZBO 2 & Nint an n n & प्राप & 3 & $570 \mathrm{Ch}$ & $\sqrt{A+5}$ \\
\hline 2802 & 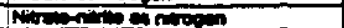 & vat & Ju & 530 & Nin \\
\hline 2032 & 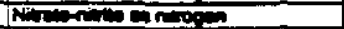 & Wa & & seotuen & ANis \\
\hline 2802 & Nition as nivom & Bus & $\sqrt{0}$ & Soluce & $\sqrt{N_{1}}$ \\
\hline 2002 & 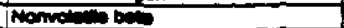 & एक & $\tilde{u}$ & $1 . 0 \longdiv { P C }$ & ANT \\
\hline 2802 & 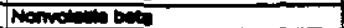 & wa & $\bar{u}$ & $17.87 \overline{C C}$ & ANDS \\
\hline 2862 & 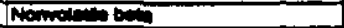 & वक्य & $\mathbf{U}$ & $0.01 \bar{C}$ & ANCS \\
\hline 2003 & 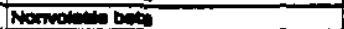 & vain & U & $1 . 3 \longdiv { F C }$ & TANBS \\
\hline 2802 & Nompin Dos & Taria & & $9.45 \mathrm{FCI}$ & ANB \\
\hline 2802 & pen & Thin & IJ & $5.37 \mathrm{Pm}$ & ANES \\
\hline ZEO 2 & Pat & owses & J & 5.30 PW & ANDS \\
\hline 2862 & pan & Wans & J & $5.51 \mathrm{PH}$ & ANOS \\
\hline ZBG 2 & phy & Mas & Is & 5.55 PA & ANCS \\
\hline ZEG 2 & Potansiam-10 & Wos & क्ष & $-14.7 P C$ & ANOS \\
\hline$\overline{Z E G} 2$ & Potatrixim-10 & ares & W & $2.45 \mathrm{PCL}$ & ANSS \\
\hline 2062 & Potentan-10 & $\cos 4$ & Ui & $32.6, \mathrm{PCL}$ & ANDS \\
\hline ZEG 2 & Potensivin-10 & 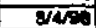 & & $39.52 \mid \overline{P C L}$ & ANES \\
\hline
\end{tabular}

WSRC-RP-99-00002 
WSRC-RP-99-00002

\begin{tabular}{|c|c|c|c|c|c|c|}
\hline WES & iANaLrTE & DATE & QUAUFIER & RESULT & JUNT & IFOAMAT \\
\hline 2802 & Promentarmis 14 & vasis & $R$ & $7 \overline{82}$ & Pa & ANDS \\
\hline 2962 & Promenten-144 & vatio & U & .1 .9 & IFCL & ANBs \\
\hline 2862 & 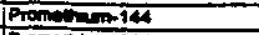 & vara & U1 & 0.24 & PCL & ANOS \\
\hline ZEG 2 & Promeniming & vain & in & 0.81 & BPC & ANOS \\
\hline EEG 2 & Pronention 120 & pwis & Ut & .3 .2 & $A C L$ & ANOS \\
\hline 2002 & Promevien-146 & Man & $\bar{y}$ & -153 & $P C$ & ANOS \\
\hline 2802 & Promentim 140 & gare & U & 1.72 & PCA & ANDS \\
\hline EQ6? & Promericint 14 & 840 & W & 3.5 & FC & ANOS \\
\hline ZEG 2 & 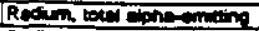 & 040 & U⿺辶 & 0.10 & $P a$ & ANOS \\
\hline ZEG 2 & 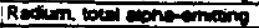 & बare & $\mathbf{u}$ & 021 & FCL & ANOS \\
\hline ZEG 2 & 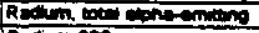 & onst & Iu & 0.3 & PCA & NAS \\
\hline ZEG 1 & Recinin-2x & g/40 & & 0.50 & PCL & $\longdiv { A N O S }$ \\
\hline 2902 & A $=\cos -220$ & ows: & UI & 0.16 & $P C 2$ & ANDS \\
\hline 2002 & Rechan-223 & 040 & Uit & 0.34 & $P C$ & ANES \\
\hline 2062 & Recium-230 & vars & UI & 043 & $P C$ & ANBS \\
\hline 2802 & Recimm-220 & Ojan: & & 1.4 & PCi & ANes \\
\hline 2062 & Rechinge & Qwas & & 167 & PCL & $\operatorname{Nis}$ \\
\hline ZEG 2 & $R=-1 m-228$ & $\operatorname{las}$ & w & -0.33 & $P C$ & ANS \\
\hline 2862 & Rectem-2al & W/is & $\mathbf{G}$ & 0.55 & PCa & $A \operatorname{Sis}$ \\
\hline 2862 & Resen-22 & y4at & $\mathbf{0}$ & 0.837 & $P C$ & ANBS \\
\hline 2802 & $\operatorname{Rec} \ln 22$ & पivin & $\mathbf{u}^{\mathbf{n}}$ & 239 & PCL & $A \times B$ \\
\hline 2002 & Rectem-2in & 840 & & 0.097 & DC & ANeS \\
\hline$\overline{802}$ & Rutenimentes & ens & $\mathbf{u}$ & -29.76 & A & Anes \\
\hline ZEG 2 & Rethenentos & Ma & $\mathbf{u}$ &.+16.75 & FCa & ANis \\
\hline 2002 & Ruthinim 100 & Bast & U & -15.55 & $\overline{F C}$ & ANis \\
\hline 2002 & 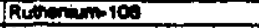 & ysen & $\mathbf{M}$ & 9.03 & $P C$ & ANDS \\
\hline 2002 & 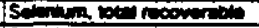 & 8 & $\mathbf{U}$ & 5) & Uद: & ANS \\
\hline 2032 & 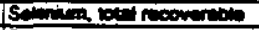 & $\operatorname{sen}$ & U & 80 & IUa & ANo \\
\hline 202 & 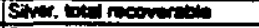 & Eas & $\mathbf{U}$ & 0.54 & Ua & Ans \\
\hline 2862 & 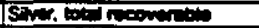 & Q14: & $\mathbf{U}$ & 7 & Ua & An:S \\
\hline 2802 & 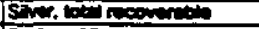 & Qwis & $\mathbf{u}$ & 3 & Ua: & ANDS \\
\hline ZEG 2 & $50 \sin 22$ & बin & Uu & -1.02 & Fa & ANeS \\
\hline $2 E 02$ & $50-\min 2 \pi$ & $8 / 5$ & $\mathbf{u}$ & 0.54 & IPC & ANS \\
\hline 2062 & Sosinga & gents & फ़ & 1.36 & PCC & $\mathrm{ANS}$ \\
\hline ZQ62 & Solinga & 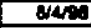 & $\mathbf{U}$ & 2.40 & FCL & ANis \\
\hline 2062 & Textrame & Waris & $\mathbf{U}$ & -2.2 & $F$ & ALS \\
\hline ZEG 2 & Tectingen & Wwin & $\bar{U}$ & -1.01 & $\overline{P C}$ & AN: \\
\hline 202 & Texnomen & Wa: & U一 & -0.73 & $\overline{a c}$ & $A N=3$ \\
\hline EO2 & Tectuminace & wein & $\mathbf{U}$ & 0.502 & $\overline{C L}$ & ANES \\
\hline 2832 & Toctwienes & $84 \times$ & $\overline{\mathbf{\omega}}$ & 3.5 & FCL & aNis \\
\hline 2802 & 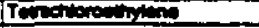 & owise & $\mathbf{U}$ & 1 & IUt & Ares \\
\hline 2802 & Tetocteromining & Jaris & $\mathbf{U}$ & & पe & ANS \\
\hline 2002 & 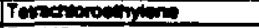 & Qwas & $\mathbf{W}$ & 5 & Ua & ANes \\
\hline 2802 & $\sqrt{7+113}$ & ansis & IU & -3.30 & DC & ANes \\
\hline 2002 & $\operatorname{Tan} 113$ & Bares & IN & 0.04 & $P C_{1}$ & $A N=3$ \\
\hline 2002 & $\operatorname{Tin} 113$ & एकas: & H & 0.20 & $\overline{P C L}$ & ANES \\
\hline ZEO 2 & Folente & 8W: & $\mathbf{u}$ & 1 & Ual & ants \\
\hline 2802 & Tohm & owas & $\mathbf{u}$ & S. & Ua & ANis \\
\hline 2862 & Tohine & gare & $\boldsymbol{\omega}$ & & iua & ANO \\
\hline $2 B$ Z 2 $_{2}$ & 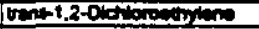 & owe & U & & UGL & $A \times B$ \\
\hline 2862 & 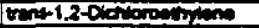 & $24 \sqrt{20}$ & U & 3) & Uat & ANS \\
\hline 2862 & 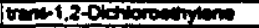 & Wases & w & 5 & Ua & ANB \\
\hline ZEO 2 & 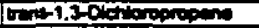 & Wes & U & I & UEL & ANDS \\
\hline $2 \mathrm{ES2}$ & 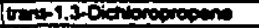 & Q 140 & IU & & Ua & AN: \\
\hline ZEO 2 & 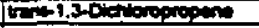 & arses & & 5) & UEL & ANes \\
\hline 2862 & 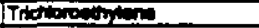 & $8 w$ & (U) & 1 & UEL & AMS \\
\hline 2802 & 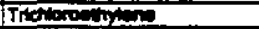 & gares & u & S: & UQ & ANis \\
\hline 2802 & 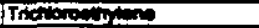 & $\cos$ & W & & पE & ANis \\
\hline 2802 & 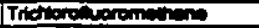 & wan & $\mathbf{U}$ & 1 & Ua & ANS \\
\hline 2802 & Tide & Qwa & 0 & 5 & ua & ANE \\
\hline 2503 & 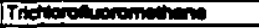 & Q6/2 & $\omega$ & $\mathbf{5}$ & Ua & AN:S \\
\hline 2062 & $x y+a n$ & Wain & U & 5] & प्C & $\sqrt{\text { ANOS }}$ \\
\hline 2862 & $x$ inenes & Wक & $\boldsymbol{\omega}$ & & Uat & TANBS \\
\hline 2002 & Yothengen & 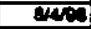 & $\bar{R}$ & 3.23 & $\overline{C L}$ & ANES \\
\hline 2002 & Ywownes & vais & U & -3.03 & PCa & IANes \\
\hline ZEG 2 & rotones & 8/40 & $\mathbf{U}$ & -1.44 & 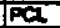 & ANS \\
\hline 2002 & roventes & 8W: & U & 0.20 & FC & 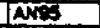 \\
\hline 2802 & $2 \ln 6$ & van & प्. & -6.94 & IFC & AN \\
\hline 2002 & 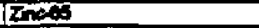 & Q65: & $\mathbf{u}$ & 0.79 & IPC & Ans \\
\hline 2832 & Indes & Q⿻: & I & .2 .08 & BFC & Ans \\
\hline 2802 & $\min x$ & BW/2 & $\mathbf{U}$ & -0.10 & $1 P A$ & Ans \\
\hline 2EB 2 & 2 won & When & u & -1.53 & $P C$ & Ans \\
\hline 2602 & $2 \cos 10$ & क्w & $\mathbf{U}$ & 0.75 & $F C$ & ANDS \\
\hline \multirow[t]{2}{*}{2002} & $2 \operatorname{con}=0$ & gave & $\mathbf{u}$ & 6.50 & FC & ANDS \\
\hline & & & & & & $\cdots$ \\
\hline EEG 1 & 1.1.1-Thateroenters & $1 / 3 \times 1$ & U & & Ua & ANoS \\
\hline 2801 & $1,1.1 .7$ notionocinase & $1 / 30 \%$ & iv & & 10a & ANBS \\
\hline 2061 & 1.1.2.2. Torachionoentionse & 1305 & U & & vel & ANS \\
\hline 2001 & 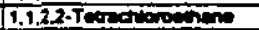 & $1 / 300$ & I0 & & iva & ANS \\
\hline 2801 & 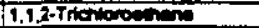 & 1300 & U & 1 & UaL & ANOS \\
\hline 2EG I & 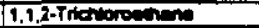 & $1 / 300$ & U & & UUG & AN:S \\
\hline $2 B G_{1}$ & 1,1-0ienlorocenterse & $1 / 3000$ & U & & Wa & ANBS \\
\hline 2061 & 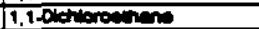 & 13000 & (i) & 5 & WE. & ANBS \\
\hline 2861 & 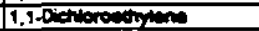 & $1 / 3000$ & $\bar{u}$ & 1 & UिG & ANos \\
\hline 2061 & 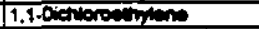 & $\sqrt{300}$ & U & S & Ua & ANBS \\
\hline 2061 & 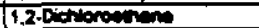 & $1 / 300$ & 0 & 1 & Uिa & ANBS \\
\hline 2001 & 1.20 detrorouthen & 1r300 & $\mathbf{u}$ & $\mathbf{5}$ & Uूa & ANOS \\
\hline 2891 & 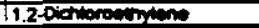 & $1 / 3000$ & tu & & ivat. & TANDS \\
\hline
\end{tabular}




\begin{tabular}{|c|c|c|c|c|c|c|}
\hline WELI & ANALVTE & DATE & OUAUFER & jRESULT: & iUंजा & FORMUT \\
\hline $2 \mathrm{BC} 1$ & 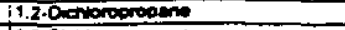 & $; 1 / 300$ & एo & & TUG & ANOS \\
\hline 2001 & 1.2 .0 icturopropent & $1 / 3000$ & 10 & & DUEL & ANOS \\
\hline$\angle B G 1$ & 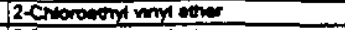 & $1 / 3000$ & $\overline{0}$ & & $\sqrt{\bar{a}}$ & ANDS \\
\hline $2 \overline{B C 1}$ & 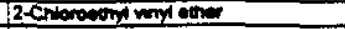 & $130 \times 0$ & v & 10 & $\sqrt{6}$ & ANos \\
\hline ZQG 1 & Acinimzen & $1 / 3000$ & $\omega$ & .1 .20 & F्C & ANOS \\
\hline 2801 & Actinin-20 & 1300 & $\overline{0}$ & 0.4 & $\overline{P C}$ & AN:S \\
\hline 2801 & Acenim 220 & 1309 & ut & 1.99 & FCL & ANES \\
\hline 2901 & Acenimin 220 & $1 / 36+2$ & uin & 11.35 & 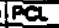 & ANPS \\
\hline 2861 & 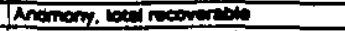 & $1 / 300$ & U. & 27. & U⿺ & ANs: \\
\hline ZEG 1 & 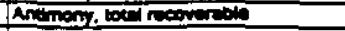 & 1300 & $\omega$ & 2.46 & पat & ANos \\
\hline ZEOT & Antinom 126 & $1 / 3000$ & जi & 0.06 & F्C & ANOS \\
\hline ZEG 1 & Antimern 124 & $1 / 30 \mathrm{D}$ & G & 0.30 & PCL & ANBS \\
\hline 206 1 & Animang 124 & 1300 & $\bar{G}$ & 0.02 & Fa & ANBS \\
\hline ZEG 1 & Antinem 125 & 1300 & ut & $-2.32 !$ & IE & ANOS \\
\hline 2861 & Antinony-125 & $1 \mathrm{son}$ & III & .1 .11 & $A$ & MNS \\
\hline 2861 & Antiming 125 & 130\% & Ui & 0.80 & $A$ & ans \\
\hline 261 & Antingorit 125 & $1 / 3000$ & Ui & 3.78 & मिल & ANes \\
\hline 2001 & 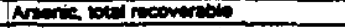 & $130 \%$ & U & & twa & ANAS \\
\hline 2001 & Anerie wed neoperdis & $1 / 300$ & $\underline{0}$ & 40 & to & ANES \\
\hline 2001 & 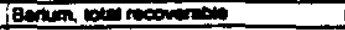 & $1 / 30 \mathrm{Di}$ & & 9 & फ्ट & ANDS \\
\hline 2001 & 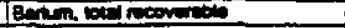 & 1300 & & 2.01 & पिए & ANCS \\
\hline 2861 & 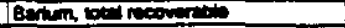 & $1 / 30 \mathrm{D}$ & & 9.201 & I0工s & ANS \\
\hline 2861 & Bostini33 & $1302]$ & Tि & .1 .7 & AC & ANiS \\
\hline 2861 & $6 \operatorname{lng}_{13}$ & 1300 & $\omega$ & 1.32 & Aa & Anis \\
\hline Z6! & Benter-133 & 1/300 & (u) & 2.73 & $\overline{A C}$ & ANis \\
\hline ZEO 1 & (1) & $1 / 300$ & U & & Wa & Ans \\
\hline$\overline{\mathrm{ZBO} !}$ & 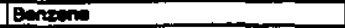 & $1 / 300$ & 0 & & (1) & ANis \\
\hline 2001 & 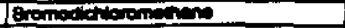 & 130ive & to & & tक: & ANDS \\
\hline 2001 & 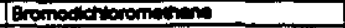 & 150ial & IU & s) & $\sqrt{1+a}$ & ANES \\
\hline 2001 & Bromenom & $130 \mathrm{R}=1$ & IU & & Wa: & ANes \\
\hline 2001 & Broctionis & t/sand & U & S) & क्ये & ANos \\
\hline 201 & 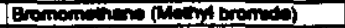 & 1300: & u & T & wa & AN:S \\
\hline एक & 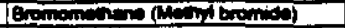 & 130: & 0 & 10 & प्त & Anis \\
\hline 2001 & 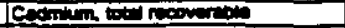 & 1/30id & एu & 4.7 & पa & ANis \\
\hline Z801 & 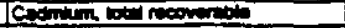 & 1/30k & U & 3) & tus & ANis \\
\hline 2891 & 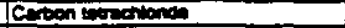 & 1300 & iv & & प्a & ANES \\
\hline 2001 & 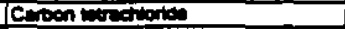 & 1305 & iv & & पa & AMOS \\
\hline 2001 & Cententa & $1 / 300$ & $\overline{\mathbf{T}}$ & .14 .18 & B & An: \\
\hline 2901 & Conemis & $3 / 300$ & प्⿱ & 0.57 & PC & ANCS \\
\hline 201 & Comeris & $1 / 30 \mathrm{sin}$ & U & 1.09 & Fat & TASE \\
\hline 2001 & Centina & Trong & ut & 0.12 & Pa & $A N B$ \\
\hline 2001 & Centing & 130\% & u & .3 .03 & Da & ANS \\
\hline 001 & $C A=134$ & 1/30: & $\mathbf{U}$ & $\mathbf{3 . 6 1}$ & Ea & AN: \\
\hline $2=01$ & Cen & $1 / 30=$ & $\mathbf{T}$ & $-1,6$ & EC & ANS \\
\hline 2061 & Centoris & 1300 & $\overline{\mathbf{u}}$ & 0.45 & $\mathrm{AC}$ & ANS \\
\hline $\mathrm{ZOO} 1$ & Cosing 137 & 1300 & TI & .2 .07 & DC & ANDS \\
\hline 290: & Ce: & Irsori & $\overline{\mathbf{u}}$ & 0.54 & Ea & A: \\
\hline 2001 & Cesing-137 & 1500 & $\overline{\mathbf{\omega}}$ & 0.0 & $E$ & Akis \\
\hline 2801 & Cenim 137 & $130=$ & $\overline{\mathbf{T}}$ & 1.54 & E & $A \mathrm{~N}^{3}$ \\
\hline ZEG 1 & Criorobinam & 1300 & $\overline{0}$ & 1 & Wa: & ANis \\
\hline 2801 & 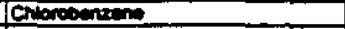 & 1/300/ & 0 & 5 & UदC & ANSS \\
\hline 2861 & Chicopenters & 1300 & 0 & 1) & Uat & ANES \\
\hline ZEG 1 & Chibreanem & $1 / 300$ & IU & 10 & U⿺辶 & ANis \\
\hline 201 & 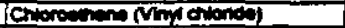 & 1scont & te & & Uिa & TANes \\
\hline ZEG! & 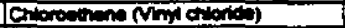 & $130 \%$ & Uu & 10 & Ua & ANoS \\
\hline ZEG T & Chigrobinn & 1rovint & U & T & $\overline{\mathbf{c}}$ & ANeS \\
\hline ZEG 1 & Chiorionen & $1 / 3000$ & 0 & 5) & va & ANis \\
\hline$\overline{\mathrm{EBG} 1}$ & 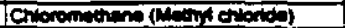 & $2 / 300$ & $\mathbf{u}$ & 1 & Ua & NNS \\
\hline ZEQ I & 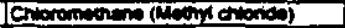 & 1300 & $\mathbf{U}$ & 10 & $0 \overline{2}$ & ANDS \\
\hline 2061 & 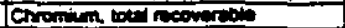 & $1 / 305$ & J & 3.01 & U⿺辶: & ANOS \\
\hline ZEG 1 & 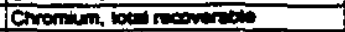 & $100 \times$ & U & 5 & U⿳亠二口⿱一⿻上丨 & TAN'S \\
\hline 2801 & 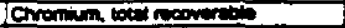 & 1300 & $\tilde{u}$ & 7 & Iúa & AN:S \\
\hline ZEO 1 & 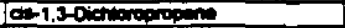 & $1 / 30 \mathrm{n}$ & U & 11 & Jua & ANB \\
\hline ZEO I & 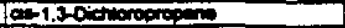 & 1/300in & $\mathbf{U}$ & S) & ज्ञ & MNB \\
\hline 201 & $\cos ^{2}-57$ & $1 / 300$ & U & 0.540 & $\overrightarrow{\alpha a}$ & AN:S \\
\hline 2861 & teosens 57 & 1300en & $\overline{\mathrm{L}}$ & 0.20 & Ea & An:B \\
\hline 2061 & $\cos 257$ & 130is & $\overline{\mathbf{\omega}}$ & 0.0. & FC1 & tanis \\
\hline 2001 & $\cos 257$ & 1500 & $\mathbf{\omega}$ & 0.5 & $\bar{a}$ & AYS \\
\hline ZEC 1 & Cosens & $1 / 302$ & 0 & -2.00 & a & An: \\
\hline 2001 & cosens & 1300 & (L) & .1 .32 & IFC & ANES \\
\hline 2061 & $\cos 25$ & 1300 & $\mathbb{0}$ & 0.01 & Fa & 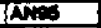 \\
\hline 29.61 & $\cos 20$ & 130 & $\mathbf{L}$ & -1.00 & $\bar{a}$ & NE: \\
\hline 2801 & $\cos \theta x$ & 1/501 & O & 0.00 & F्ञ & A. \\
\hline 2031 & cosing & $1 / 30,1$ & U & 0.33 & $\overline{F C}$ & AN \\
\hline 2001 & $\cos \theta$ & $1 / 50$ & (1) & 0.002 & Pa & ANis \\
\hline $\mathrm{ZEO}:$ & 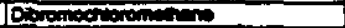 & 1300 & D & 9 & प्र & AN:D \\
\hline 2001 & 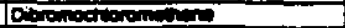 & 1300 & 10 & 5) & Ua & Ants \\
\hline 2001 & 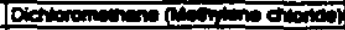 & 1500 & $\overline{0}$ & 3.34 & पa & Ans \\
\hline 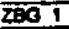 & 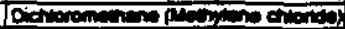 & 150 & 0 & 1.04) & $\sqrt{02}$ & ANS \\
\hline $\mathrm{ZEO} 1$ & 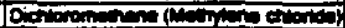 & $1 \mathrm{sen}$ & $\overline{0}$ & 5.201 & ive & $A W^{3}$ \\
\hline 2001 & 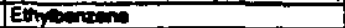 & 130 & tu & Tा & WE & ANtS \\
\hline 2001 & 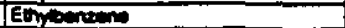 & T/300 & 10 & 5) & Lat & ANis \\
\hline 2801 & Exropiem is2 & trang & $\mathbf{0}$ & .1 .71 & PC1 & ANC: \\
\hline ZEO? & Eurcepin-152 & $1 / 300$ & $\mathbf{U}$ & 0.400 & FCh & ANES \\
\hline ZEG I & Evopien 152 & 1/30\% & $\mathbf{W}$ & 12.62 & $\overline{F C}$ & anis \\
\hline 2801 & Eloponim-152 & 1/300:0] & IUT & 13.55 & Fa & AnOS \\
\hline 2061 & Evooumisa & I/30ria! & UT & .2 .92 & a & Anis \\
\hline 2001 & Expoum-1S4 & T30\% & ui & 0.904 & 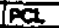 & ancs \\
\hline
\end{tabular}

WSRC-RP-99-00002 


\begin{tabular}{|c|c|c|c|c|c|c|}
\hline WELL & ANALVTE & OATE & QUAUIFIER & RESULT & IUNAT & FORMAT \\
\hline $2 B G 1$ & Euroum is & $1 / 30 \sqrt{9} 0$ & iv & & $P C 1$ & ANOS \\
\hline $2 \bar{B} 1$ & Evecoum-154 & 1/3050 & Ui & 3.33 & FA & anss \\
\hline 2869 & Eveopintiss & $1 / 309$ & U & .2 .21 & IPC & ANES \\
\hline 2001 & Europents 15 & 7300 & UT & 2.00 & $P C$ & ANS \\
\hline $2 \mathrm{BO} 9$ & Euroginimis & t/30\% & 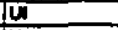 & 2.4 & FCa & ans \\
\hline 2001 & Europinen 15 & 1 क्लित्य & U & 4.93 & AC & ANDS \\
\hline YEG! & Gromentens & $1 \sqrt{300}$ & & 129 & $A C$ & ANOS \\
\hline 2001 & 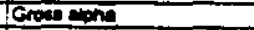 & $1 / 3000$ & & 2.33 & $P$ & ANBS \\
\hline EQ 1 & Groat onsense & 1300 & & 2.8 & $P Q$ & ANS \\
\hline 2301 & Goesene & Tत्00 & & 3.42 & Fa & ANos \\
\hline ZEG 1 & Lead been neoveris & Tropes & $J$ & 7.3 & UTE & ANPS \\
\hline 2001 & 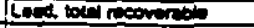 & 1/3083 & I & 7.5 & Ual & Ares \\
\hline ZES ? & 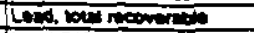 & 1500 & iv & 8.40 & ven & AN:S \\
\hline ZEO 1 & Lovot212 & isore & W & .1 .97 & Pa & ANDS \\
\hline 2801 & $\log 212$ & I/30rol & is & 0.12 & $P C$ & ANDS \\
\hline 2061 & Leser212 & isoras & $\mathrm{u}$ & 1.85 & ac & NMS \\
\hline ZEG 1 & Lect212 & 130ing & w & 5.40 & FC & ANCS \\
\hline 2061 & Menamiosis & 130ल0 & ivi & .1 .04 & PC & ANS \\
\hline 21 & Monation & t/300 & Ui & 0.11 & Pa & ANOS \\
\hline ZEG 1 & Mangenesis & $1 / 300$ & IU & 0.4 & $\mathrm{PCL}$ & AN:S \\
\hline ZBE 1 & Mingons & 1000 & $\bar{\omega}$ & 7.5. & pa & $A N B$ \\
\hline 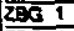 & 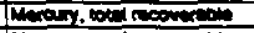 & Trasin & U & 0.2 & va & ats \\
\hline 2901 & 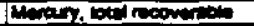 & 1/300 & U & 0.7 & Ua & $\sqrt{A+3}$ \\
\hline 2E0 1 & 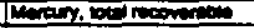 & 130: & U & 1 & ज्ञn & ANB \\
\hline 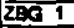 & 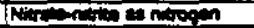 & $4 / 3 \% \bar{a}$ & & (154) & प्व & $A \times C$ \\
\hline 2861 & 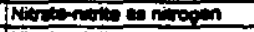 & 1/30\% & & 180 & IU⿺ & ANeS \\
\hline$\overline{Z B O} 1$ & 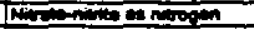 & $1 / 3000$ & & 1350 & Ua & ANOS \\
\hline 2801 & 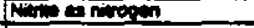 & $1 / 300$ & $W$ & 20 & UूC & No6 \\
\hline 2861 & 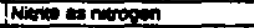 & $1 / 3000$ & UR & 5 & wat & AN:B \\
\hline EQ 1 & 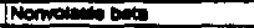 & 1/30in & $J$ & 3.30 & $\overline{P C}$ & Ans \\
\hline 2061 & 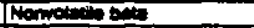 & 1/3000 & $\sqrt{3}$ & 4.10 & Fa & ANS \\
\hline 2801 & 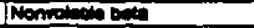 & $1 / 300$ & J & 9.39 & $\bar{F}$ & Ans \\
\hline 2001 & Homotion Dese & $1 / 3000$ & & 5.22 & PC & Nis \\
\hline 2001 & T敕 & 130 is & $\mathrm{J}$ & 5.14 & Fin & AN:S \\
\hline $20 \overline{1}$ & in & $1 / 300$ & $\sqrt{3}$ & 5.22 & $\overrightarrow{F+1}$ & ANS \\
\hline 2891 & pan & $1 / 3020$ & I & 5.53 & $\overline{P H}$ & $\sqrt{A \times C S}$ \\
\hline 2801 & on & isons & J & 5.9 & $\vec{F}$ & ANo \\
\hline 2001 & $P Q=0$ innmo & 1300 & UT & .12 .00 & FE & $A$ \\
\hline 2001 & Potionamas & $1 / 30 \times$ & $\mathbf{U}$ & 124 & Fa & AN: \\
\hline 2801 & Potarionero & T/3as] & U & 59.37 & FCL & Ans \\
\hline 2069 & Pod & 150\% & Uي & 2.34 & FC & ANis \\
\hline 2001 & Promenimin & $1 / 30 ;$ & U & .2 .75 & a & ANS \\
\hline 2801 & Promberma & $1 / 300$ & u & 0.30 & FCL & $\operatorname{ANB}$ \\
\hline 2001 & Promentiom ist & 1300 & (4 & $0.5 \%$ & Pa & ANCS \\
\hline 201 & Promeniming 14 & $1 / 300$ & $\overline{\mathbf{u}}$ & 4.59 & FQ & ANe \\
\hline 2001 & 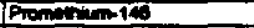 & $1 \sqrt{30}$ & $\mathbf{u}$ & 80 & Fa & Nis \\
\hline 2099 & Frominging 146 & rison & $\mathbf{s}$ & 0.83 & Ea & ats \\
\hline 2831 & Promeniming 19 & $1 / 3000$ & $\bar{M}$ & 3.25 & FE & $A N=$ \\
\hline 2801 & Promenisin 140 & 13000 & $\mathbf{W}$ & 4.15 & IFC & AN:S \\
\hline EQG I & 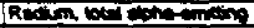 & irso:ia & U. & 0.23 & FQ & $\sqrt{\operatorname{ans}}$ \\
\hline 2801 & 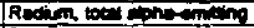 & $1 / 30 \mathrm{D}$ & U & 0.04 & FEt & $\min$ \\
\hline 2001 & 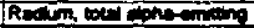 & 1300 & & 0.20 & 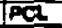 & NAS \\
\hline ZEG 1 & 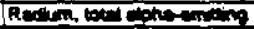 & 10300: & & 0.6 & PQ & ans \\
\hline 2001 & 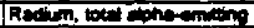 & $130 \mathrm{O}$ & & 0.7 & PCL & No: \\
\hline 2069 & Reciun-228 & 1300 & J & 0.67 & PC2 & 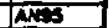 \\
\hline ZEG ? & $\operatorname{Ron}=220$ & $9130 \mathrm{ag}$ & 5 & 0.60 & FC & ANB \\
\hline$\overline{Z B O 1}$ & Aximen-230 & $430 \mathrm{Nol}$ & (vin & 0.35 & FEL & $A \operatorname{MB}$ \\
\hline ZEG 1 & $R=\ln 220$ & $1 / 30 ; 0$ & W & 0.430 & PCI & ANES \\
\hline 2801 & IRedingen & roon: & $\mathbf{u}$ & 0.25 & $P C$ & ANes \\
\hline 2861 & Rnominge & Tिक्या & u & 0.00 & $F$ & AN \\
\hline$\angle B O 1$ & Recingen & $1 / 3000$ & & 4.0 & $F$ & $A+3$ \\
\hline 2969 & Rotreniontos & Forin & $\mathbf{u}$ & .20 .30 & $F E$ & AN3 \\
\hline $2 B 61$ & RLention-10 & 1300 & $\mathbf{0}$ & 4.22 & $\overline{P C}$ & AIS. \\
\hline 2001 & Ruterians & $1 / 30 ;$ & $\overline{\mathbf{\omega}}$ & 9.12 & PC & A: \\
\hline$\angle E O 1$ & Retrition 100 & 1/300i & $\bar{\omega}$ & 30.7 & FE & 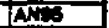 \\
\hline EBG 1 & 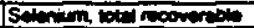 & 15ant & J & 3.95 & Uद: & ANS \\
\hline EOS 1 & 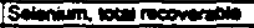 & 1/30id & U & 01 & Wa: & Nes \\
\hline 2801 & 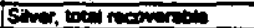 & trom & J & 0.74 & UE: & Ans \\
\hline$\angle B G 1$ & 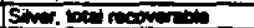 & $\operatorname{tscon}$ & J & 0.04 & U्C: & $\alpha=6$ \\
\hline 2001 & 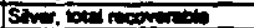 & $1 / 300$ & $\bar{U}$ & & We & $\bar{A}: 3$ \\
\hline 2061 & Sottina & $1 / 300$ & $\bar{u}$ & 0.04 & PC & ANes \\
\hline$\overline{Z E G 1}$ & $50 \sin 2$ & $\sqrt{\infty}$ & D & 0.341 & PC & $\overline{A \% 3}$ \\
\hline$\overline{2 B O} 1$ & Soding 2 & 130 & $\bar{\omega}$ & 0.30 & PC1 & $A$ \\
\hline 2001 & Sosing 2 & $1 / 300$ & U. & 0.01 & FC & An: \\
\hline $2 B G 1$ & Techente & $1 / 500$ & $\mathbf{v}$ & A.81 & IPC & ANG \\
\hline 2001 & Tecomintas & $1 / 30 \mathrm{D}$ & Uు & 2.11 & FCL & $A \times B$ \\
\hline 2801 & Tectinines & 1/300] & $\mathbf{U}$ & 3.34 & pa & ANa \\
\hline$\overline{Z \mathrm{EO} I}$ & Tectinentente & trom & U. & 8.05 & PQ & ANos \\
\hline 2001 & Tectinienes & 1300: & $\mathbf{U}$ & 8.34 & FC & ANS \\
\hline EQ: & Teroctionosinions & 13000 & U一 & & Uat & Ans \\
\hline ZEOT & 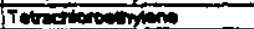 & iscons & (1) & & va & ANBS \\
\hline $2 E B 1$ & Tn-113 & 1300 & $\vec{\omega}$ & .2 .42 & $P a$ & $\sqrt{N<6}$ \\
\hline $200 \div$ & Thn.113 & 130\% & $\overrightarrow{\mathbf{U}_{1}}$ & .1 .35 & $P C$ & ANS \\
\hline 2051 & Thm113 & isoral & $\mathbf{U}$ & 0.22 & Q & Ans \\
\hline$Z \overline{Z O 1}$ & Tolente & 13000 & $\mathbf{U}$ & & UG & An: \\
\hline ZEG 1 & Totions & isonst & U & 5 & Ua & AN: \\
\hline 2861 & 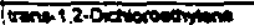 & tasorat & $\mathbf{U}$ & 1: & va & AKSS \\
\hline 286 & trent-1,3-0ionorogropents & 1300 & U & & UGL & ANis \\
\hline
\end{tabular}

WSRC-RP-99-00002

I-11 


\begin{tabular}{|c|c|c|c|c|c|}
\hline WELL & ANALYTE & DATE & OUALIFIER & RESULT IUNIT & FORMAT \\
\hline 2961 & 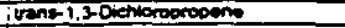 & $1 / 30 \sqrt{90}$ & IU & SIUG & AN9S \\
\hline 2861 & 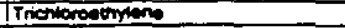 & $1 / 30 \sqrt{20}$ & Uu & SUG & ANOS \\
\hline $2 E G_{1}$ & IThenoromentione & $1 / 3090$ & Wu & IUGG & ANDS \\
\hline 2061 & 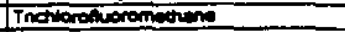 & 1300 & iv & IU⿴囗十 & IANSS \\
\hline $2 B G 1$ & 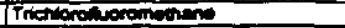 & $1 / 3050$ & iu & SणGL & ANSS \\
\hline 2861 & xymans & $1 / 309$ & U. & Siva & ANOS \\
\hline $296+$ & rotentes & 1 1700 & us & $-2.22 \mid P C L$ & ANBS \\
\hline $2 \mathrm{BO}$ & Yotones & $1 / 300$ & I9 & $0.2751 P C$ & ANES \\
\hline$\angle B C 1$ & Trowimen & $1 / 30 \times$ & (U) & $0 . \% 1 P C L$ & $\triangle N O S$ \\
\hline ZEG 1 & Yowingers? & $1 / 300$ & UI & $1 . \infty \mathrm{PCL}$ & ANOS \\
\hline 2961 & zineds & $1 / 300$ & wa & Q.17)PCL & ANDS \\
\hline $2 B 69$ & Zneos & $1 / 200$ & 여 & $-2.33 P C 1$ & $\sqrt{A N B S}$ \\
\hline $2 B G 1$ & Zneas & $130 \%$ & in & $.1 .7 / P C L$ & ANG \\
\hline 2601 & Zineas & 1309 & Uu & $.1 .34 \mathrm{FCL}$ & ANOS \\
\hline 2861 & Zreonim-05 & t/30is & जi & $.2 .10 / P C L$ & ANS \\
\hline 2061 & Zreonimis & 1/30\% & $\bar{v}$ & 29PC & $A N 5$ \\
\hline 2861 & Ziromings & $1 / 30 \times$ & It] & $5.28 \mathrm{BC}$ & Arits \\
\hline 286 iA & 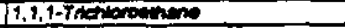 & 2200 & iv & s) & ANiS \\
\hline $2001 \mathrm{~A}$ & 1,1,2.2-Terectionothere & $22 \%$ & $\mathbf{U}$ & SUG & ANDS \\
\hline 206 IA $^{2}$ & 1.1.2. Therloromenten & $2 \sqrt{29}$ & I0 & Sण्र & ANDS \\
\hline $2 E G 1 A$ & $1,1+0$ chioromenese & $2 \sqrt{2} \times$ & IU & काण्C & ANDS \\
\hline 286 3A & $11.1-0$ chioroentime & $2 \sqrt{2 \pi}$ & U & 5 पिद & An:B \\
\hline $2 B O B$ & 1.2 -Dichorounters & $2 \sqrt{25}$ & IU & 5) & $A N \times B$ \\
\hline 290 & 1,2-Dediorom mime & $2 \sqrt{2}$ & iv & 5 & ANCS \\
\hline $2 \mathrm{EO}$ iA & 1.2-0idiorectionen & $2 \sqrt{230}$ & IU & Syal & anis \\
\hline $2001 \mathrm{~A}$ & $2-c_{10}$ & $2 \sqrt{25}$ & U & 10IUG & ans? \\
\hline $20 G 1 A$ & Actinterm 220 & $2 \sqrt{2 \pi}$ & UT & $-2.8 \mid \mathrm{PC}$ & ANDS \\
\hline 200 iA & $A \operatorname{con}(\min -2)$ & $2 \sqrt{250}$ & W & $2 . 4 0 \longdiv { P Q }$ & ANBS \\
\hline 28019 & Andinom 124 & 2250 & Tu & $.101 P a$ & ANOS \\
\hline 286 1A & Antrion 124 & $22 \%$ & iv & $1.301 \mathrm{FC}$ & ANBS \\
\hline ZEG 1A & Axtingon-t23 & $2 \sqrt{2 x}$ & U & $-2.20 \mathrm{PCL}$ & DAS \\
\hline $2 \overline{B O A}$ & ANenom 125 & $2 \sqrt{2 \pi}$ & IV & $9.30 P C$ & ANB \\
\hline$Z E 0$ 1A & $8=x-133$ & 2250 & IU⿴囗十 & $0.13 \overline{F C}$ & ANiS \\
\hline $2001 \overline{1}$ & Bantimis3 & $2 \sqrt{25}$ & u & $0.601 \times C$ & ANeS \\
\hline $206+\lambda$ & tomenten & $2 \sqrt{20}$ & U & 5Ua & ANS \\
\hline $286 \sqrt{A}$ & 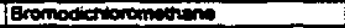 & $2 \sqrt{200}$ & E & 5ण्GL & ANS \\
\hline 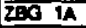 & Erenoiom & 2200 & U & SUGL & ANDS \\
\hline 283 ia & 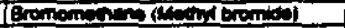 & 2250 & 0 & lofuet & TAMES \\
\hline $200 \sqrt{A}$ & 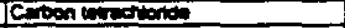 & $\sqrt[220]{20}$ & U & 5)a & ANBS \\
\hline ZEG 1A & Contentas & $\sqrt{2}=$ & U & $.7 \mid P C A$ & ands \\
\hline 2969 & Conimist & 2क्2 & (U) & $.1 .23 P C$ & IANS \\
\hline 200 & Coningmis & $22 \sqrt{20}$ & $\mathbf{u}$ & $0.7917 C$ & AN: \\
\hline $2801 / 4$ & Centering & $2 \sqrt{20}$ & (u & $1.23 / \overline{C C}$ & An: 3 \\
\hline 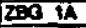 & Centim 137 & $2 \sqrt{2}$ & IU & $0.76 \overline{\mathrm{Ca}}$ & ANOS \\
\hline $2 E O \bar{A}$ & Cotin-137 & 2210 & IU & 2.J7नC & AN"S \\
\hline 2003 & Cripiodenten & $2 \sqrt[250]{20}$ & (u & 5) & Ams \\
\hline$\angle E B \overline{1 A}$ & Cronestere & $2 \sqrt{25}$ & 10 & 10, UEL & 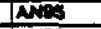 \\
\hline $200 \pi$ & 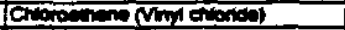 & $2 \sqrt{25}$ & I0 & व०ण0 & ands \\
\hline ZBG $\overline{\text { IA }}$ & Criponm & 225 & Iu & SUंद्य & AN: \\
\hline $200 \mathrm{~T}$ & 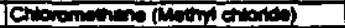 & $22 \%$ & (1) & 10UCL & An: 3 \\
\hline $2 B G \sqrt{A}$ & 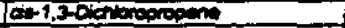 & 200 & U & 5Ua & ANS \\
\hline $286 \sqrt{A}$ & $\cos =37$ & 2250 & u & $.1 .017 a$ & ANES \\
\hline $28 \mathrm{G} \overline{\mathrm{A}}$ & Coper.57 & $2 \sqrt{200}$ & U: & $0.37 P C$ & AN: \\
\hline EQG & $\cos \cos ^{2}$ & $2 \sqrt{2 \pi 0}$ & जi & $4 \sqrt{2} \overline{P C}$ & ANiS \\
\hline $280 \overline{1 A}$ & $\cos 25$ & 2525 & a & $.2 .19 \overline{P C}$ & ANis \\
\hline ZEG $\overline{1 A}$ & $\cos 20$ & $2 \sqrt{2 \pi}$ & IU & $-1.09 \mathrm{CC}$ & AMes: \\
\hline $2 \mathrm{EG} \overline{\mathrm{XA}}$ & $\cos -\infty$ & 2290 & un & 1.41PCL & ANOS \\
\hline $28 \bar{G} \overline{1 A}$ & 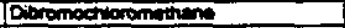 & 2220 & U. & 5UG & $A N_{3}$ \\
\hline $289 \overline{9 A}$ & 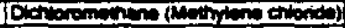 & एक्य & iv & 29,2 Ua & Ans \\
\hline $200 \overline{1 A}$ & Etromenen & $2 \sqrt{200}$ & U & SULE & ANS \\
\hline $206 \mathrm{TA}$ & Exroinin-152 & 2200 & U & .17 AIPCE & ANES \\
\hline $296 \sqrt{A}$ & Exropian 132 & $\sqrt[2 \sqrt{20}]{20}$ & $\mathbf{\omega}$ & 8.3PFC & ANS \\
\hline $296 \overline{3 A}$ & Extoping 13 & 225 & IU & $.5 . \mathrm{APC}$ & Ans \\
\hline ZEOT & Exropinis & $2 \sqrt{2 \pi}$ & U & $.1 .73 / P C$ & ANS \\
\hline $2 B D \overline{1 A}$ & Evropinm 15 & $2 \sqrt{210}$ & 4 & $3.51 \overline{P C}$ & ANes \\
\hline $2 \mathrm{EG} \overline{\mathrm{TA}}$ & Evopioniss & 202 & Tr & 0.00 & ANis \\
\hline $206 \overline{1 A}$ & Grous ations & $2 \sqrt{20}$ & & O3.3FCL & Ans \\
\hline $2001 \mathrm{~A}$ & Going and & 225 & & $97.7 \mathrm{PC}$ & ANS \\
\hline $28 B \sqrt{A}$ & Lent212 & 25 & U1 & $2.27 \overline{P C}$ & AN 6 \\
\hline ZEG $1 A$ & tones212 & 225 & (1) & 8.41FC & ANis \\
\hline $2607 \overline{14}$ & $\log _{2}$ & $2 \pi$ & (1) & -200 C & ANes \\
\hline $286 \sqrt{A}$ & Merimans & 22 & iu & $\therefore$ 1.PC & ANes \\
\hline ZEG $1 \mathrm{~A}$ & $4 \ln 20$ & 2520 & tu & $300.4 \sqrt{2}$ & ANE \\
\hline$2 8 0 \longdiv { 1 A }$ & 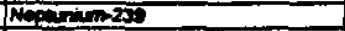 & 2हलं & ب & ग515:FC: & Ans \\
\hline EEO $1 \mathrm{~A}$ & Nin & $2 \sqrt{25}$ & & कण्य & ANis \\
\hline $2001 \mathrm{~A}$ & 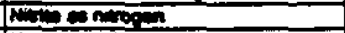 & $2 \pi \omega$ & $\omega$ & 2 प्द्र & ans \\
\hline$\overline{E B}$ iA & 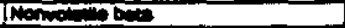 & 225 & & 41.84PC & ANB \\
\hline 200 & Hoinetion & $2 \sqrt{25}$ & & $52.50 / \bar{Q}$ & ANS \\
\hline $2861 A$ & क्ष & $2 \sqrt{2}$ & 1 & $0.201 \mathrm{PW}$ & AN3 \\
\hline $2861 \mathrm{~A}$ & Potengondo & $2 \sqrt{2 i 2}$ & $\bar{\omega}$ & $.22 .61 \mathrm{PC}$ & ANBS \\
\hline 20074 & 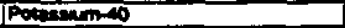 & $2 \pi=$ & (6) & $2.13 \mathrm{FC}$ & anse \\
\hline ZEG TA & Promerionstiat & $22 \pi$ & U & $\because .4 P C$ & ANES \\
\hline 296 1A & Promerinom-144 & 2200 & IT & $1.35 \ldots$ & ANDS \\
\hline 200 iA & Promeniments & 2200 & tor & -2.00 & ANOS \\
\hline $2861 \mathrm{~A}$ & Promeriment-146 & $22 \%$ & W & $4.50 / \mathrm{PC}_{2}$ & ANDS \\
\hline ZEG 1A & 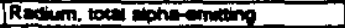 & $2 \sqrt{200}$ & & $55.51 \mathrm{PC}$ & ANOS \\
\hline ZEG IA & $R=14 m-220$ & $22 \%$ & & $7.78 \mathrm{PC}$ & IANDS \\
\hline \begin{tabular}{|l|l|} 
ZEG & IA \\
\end{tabular} & IR=oinen-220 & 22290 & & $0.02 A C$ & ANOS \\
\hline
\end{tabular}

WSRC-RP-99-00002

I-12 


\begin{tabular}{|c|c|c|c|c|c|c|}
\hline WELL & ANALYTE & OATE & QUALFIER & IRESULT & IUNT & Format \\
\hline$\overline{B B G} \overline{1 A}$ & Rustoment 100 & $2 \sqrt{200}$ & U & .1 .28 & PCL & IANAS \\
\hline ZEG $1 A$ & Rotonam 100 & 225 & W & $\$ 2.65$ & $\sqrt{\mathrm{FCl}}$ & ANSS \\
\hline 20014 & Soconen-22 & $2 / 20$ & $\mathbf{u}$ & $.1,40$ & IfFCL & AMOS \\
\hline 28014 & 500 inm-22 & $2 \sqrt{20}$ & U & 0.39 & ACLL & AN'S \\
\hline $2 B O T A$ & Tectiminemese & $2 \sqrt{200}$ & $\mathbf{W}$ & 10.78 & PCL & anes \\
\hline $2 B$ G & 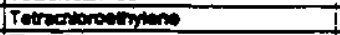 & $2 \sqrt{250}$ & $\mathbf{U}$ & & tua & ANS \\
\hline EQ 1A & 792193 & 2द्20 & WH & 4.3 & $\overline{P C L}$ & ANS \\
\hline 28014 & Trmi13 & $2 \sqrt{200}$ & W & 4.43 & PCA & Anos \\
\hline $20, \overline{1 A}$ & Tolenter & $2 \sqrt{2 \pi}$ & U & & Wua & ANDS \\
\hline 20014 & 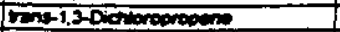 & $22 \%$ & to & & UGa & ANAS \\
\hline 28614 & 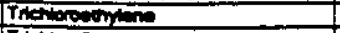 & $2 \pi=1$ & U, & & vot & ANas \\
\hline 296 iA & 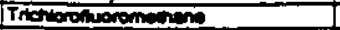 & $2 \pi=1$ & $\mathbf{U}$ & & Uि & ANCS \\
\hline ZEG $x$ & $x$ ynom & $2 / 2 \%$ & $\bar{U}$ & & Hot & ANS \\
\hline EE $1 \mathrm{~A}$ & Youme & हथ्य & W. & -1.62 & $P C i$ & ANCS \\
\hline $2 E 61 \mathrm{~A}$ & Youments & $2 \sqrt{20}$ & $\bar{u}$ & 1.4 & PC & ANis \\
\hline $260 \sqrt{4}$ & Zno-65 & 2था & $\mathbf{U}$ & .2 .14 & Pa & NAES \\
\hline 280 & Zne-os & 2220 & ज & 1.75 & FCe & $A \times B$ \\
\hline 28014 & Zroonings & $22 \times$ & $\mathbf{L}$ & .2 .52 & $\mathrm{PC}$ & ANS \\
\hline $20 G$ & 2000 in 18 & 2200 & $\mathbf{U}$ & 5.34 & $\overline{P C}$ & AN: \\
\hline 2002 & 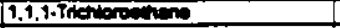 & 225 & 0 & 5 & Uिa & ANB \\
\hline $2 \overline{\mathrm{BO} 2}$ & 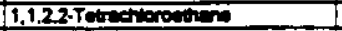 & 2250 & $\mathbf{U}$ & & Uूa & ANES \\
\hline 2862 & 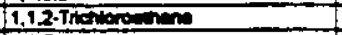 & $22 \pi$ & $\bar{U}$ & & UGL & Anis \\
\hline 2063 & 1.1 .00 onem & $2 \sqrt{2}$ & $\mathbf{u}$ & 5 & U्यू & Anits \\
\hline 2802 & $1,1-0$ a & 220 & $\mathbf{U}$ & & Ua & ANOS \\
\hline 203 & 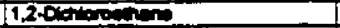 & $22=0$ & U & & Uत् & ANiS \\
\hline 2802 & $1.2+0$ id & 200 & $\bar{U}$ & & ua & ANOS \\
\hline Z6G & 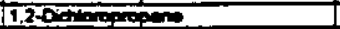 & $2 \pi 20$ & $\boldsymbol{U}$ & st & ual & ANOS \\
\hline 2002 & 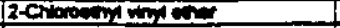 & $2 \pi \approx 0$ & $\mathbf{U}$ & 10 & UE & ANDS \\
\hline $20 \overline{2}$ & Actinin-238 & $2 \sqrt{2: 3}$ & $\mathbf{H}$ & .1 .04 & $\overline{P C}$ & $\longdiv { A N B S }$ \\
\hline 2002 & 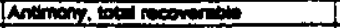 & $2 \Omega: 0$ & $\mathbf{U}$ & 27 & Ua & AN: \\
\hline $200 \overline{2}$ & Antinomin-124 & 2थ0 & $\mathbf{L}$ & .5 .64 & Fa & ANEB \\
\hline 2E: 2 & 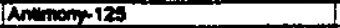 & $2 / 2=0$ & $\mathbf{U}$ & .2 .6 & PCE & ANS \\
\hline 4002 & 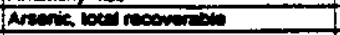 & $22 \pi$ & U & 40 & UCL & ANS \\
\hline EOS2 & 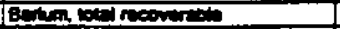 & $2 \sqrt{20}$ & & 6.1 & ual & ANas \\
\hline 2062 & $8 \operatorname{sen} 133$ & 25 & $\mathbf{U}$ & -0.21 & $P C$ & ANes \\
\hline 28G2 & onersem & 220 & $\mathbf{U}$ & 3) & Ua & ANRS \\
\hline 202 & 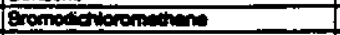 & $2 \pi 0$ & U & 5 & UCL & An:S \\
\hline 2002 & Eronevion & 20 & $\overline{\mathbf{T}}$ & 5 & ual & AN: \\
\hline 202 & 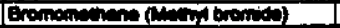 & Z2: & U & 10 & Ua & ANA \\
\hline $20 \overline{2}$ & 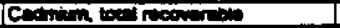 & 2था & $\bar{U}$ & 4.7 & Ua & ANGS \\
\hline $20 \overline{2}$ & 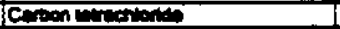 & हथा & U & 5 & Uैa & ANCS \\
\hline 202 & Conturias & $2 \sqrt{2}$ & $\mathbf{u}$ & 0.77 & FCL & ANAS \\
\hline 202 & 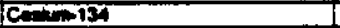 & $2 \sqrt{201}$ & $\mathbf{U}$ & 0.03 & FCL & AN: \\
\hline 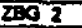 & $C=10.137$ & $2 \sqrt{2}$ & $\mathbf{T}$ & 2.42 & ACL & AN: \\
\hline 2062 & 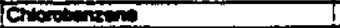 & 2द्र: & $\mathbf{U}$ & 3. & wea & Anes \\
\hline $200 \overline{2}$ & Cropomine & 2थ: & $\underline{\mathbf{U}}$ & 10 & Ua & ANC: \\
\hline 2002 & 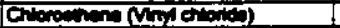 & 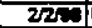 & $\mathbf{U}$ & 10 & Uen & ANDS \\
\hline 206,2 & Chorim & $22 \%$ & $\mathbf{U}$ & S & Ua & Ans: \\
\hline 8002 & 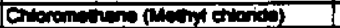 & $2 \sqrt{200}$ & $\overline{\mathbf{U}}$ & 10 & Uूa & ANES \\
\hline 2602 & 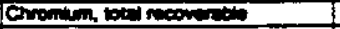 & 22:i & J & 1.5 & Ua & ANS \\
\hline 2002 & 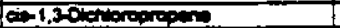 & $2 \sqrt{200}$ & $\overline{\mathbf{u}}$ & 5 & Ua & ANOS \\
\hline 2602 & Cobens? & 2य: & U. & .1 .00 & FCL & ANSB \\
\hline 2032 & Conens & 22:i & U్ & 1,00 & Fa & ANis \\
\hline $2 E 02$ & $\cos +9$ & 2200 & U. & 1.40 & PCL & $\sqrt{A \times B}$ \\
\hline 2932 & 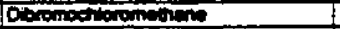 & 2थ्201 & $\mathbf{U}$ & 5. & Ua & AN:B \\
\hline 2002 & 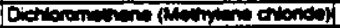 & 2820 & $\mathbf{U}$ & 29.7 & Uat & $\sqrt{A N B S}$ \\
\hline ZEG 2 & Elmporom & $2 \sqrt{2}$ & $\overline{\mathbf{U}}$ & & Uद्स & ANBS \\
\hline $28 B 2$ & Europian 152 & $2 \sqrt{200}$ & $\bar{u}$ & 11.20 & PCa & AnEs \\
\hline 2002 & Exrogitom 15A & 2200 & $\bar{T}$ & .1 .53 & FC & ANB \\
\hline 2002 & Eurciemist & 22\%: & $\overline{\mathbf{M}}$ & 0.51 & ACL & ANas \\
\hline 2962 & Grositisina & $22 \sqrt{2}$ & $\bar{u}$ & 0.70 & FCL & AN:B \\
\hline 2802 & 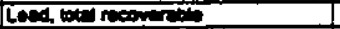 & $2 \sqrt{2} ;$ & $\mathbf{U}$ & 47 & Wa & ANB \\
\hline 2802 & LaAd-212 & $2 \sqrt{2 \pi}$ & प्र & 1.22 & FC & $A=3$ \\
\hline 2802 & worenest & $2 \sqrt{201}$ & U & 0.01 & EC & $\mathrm{AC}: \mathrm{B}$ \\
\hline $20 \overline{2}$ & 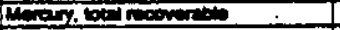 & $2 \pi \sqrt{2}$ & $\bar{v}$ & 0.0 & Ua: & AN \\
\hline$\angle B G 2$ & Nepentin-2030 & 22201 & $\mathbf{u}$ & .2150 & get & $A \times=$ \\
\hline 2802 & Non & $22 \%$ & & 7 & wa & ANis: \\
\hline 2002 & 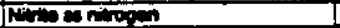 & 220 & $\mathbf{U}$ & 201 & Wa & ANG \\
\hline 2962 & Nom & 220 & $\mathbf{U}$ & 7.30 & $F$ & ANS \\
\hline $200 \overline{2}$ & pat & 220 & $\sqrt{5}$ & 5.20 & FH & Ans \\
\hline 2032 & 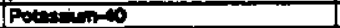 & $2 \sqrt{20}$ & $\mathbf{U}$ & 18.44 & PCL & ALS \\
\hline 2032 & 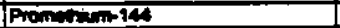 & $2 \sqrt{2}$ & $\mathbf{u}$ & 2.50 & $P C$ & ANS \\
\hline 2002 & Promenters 14 & 221 & u & .3 .00 & FE & Nes \\
\hline 2602 & 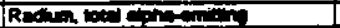 & $\sqrt{25}$ & $\overline{\mathbf{U}}$ & $\$ .37$ & FC & ANB \\
\hline $7 \mathrm{ES2}$ & 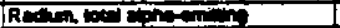 & 22 & $\mathbf{U}$ & 4.72 & $\overline{C L}$ & ANB \\
\hline $2 B \overline{2}$ & Recienzes & 225 & & 0.78 & $\overline{F C}$ & ANis \\
\hline 2802 & $R=\ln 2 x$ & $2 \pi$ & & 1.12 & PCI & ANis \\
\hline $20 \overline{2}$ & $R=0-220$ & $2 \sqrt{20}$ & $\mathbf{u}$ & .1 .23 & PC & ANS: \\
\hline 2002 & $R=1,2 a$ & $22 \pi$ & $\mathbf{U}$ & 1.70 & FC1 & ANS \\
\hline 2002 & Rutherien 100 & 2थखा & $\bar{u}$ & 5.6 & Fa & ANB \\
\hline $20 B \overline{2}$ & 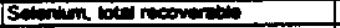 & $2 \sqrt{2}$ & $\mathbf{U}$ & 6 & Wa & ANS \\
\hline 2002 & 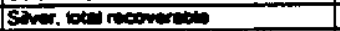 & यहi & J & 1.7 & wat & $A N=3$ \\
\hline 202 & Soctum-22 & 2का & IU & 0.57 & PCI & ANGS \\
\hline 2002 & Tectinimans & $2 \pi 01$ & $\mathbf{U}$ & 0.30 & $\overrightarrow{P C}$ & ANA \\
\hline 2002 & Tectnotionses & $2 \sqrt{23}$ & UI & 이 & PCL & ANDS \\
\hline$\overline{Z E G} \overline{2}$ & 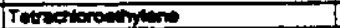 & 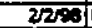 & $\mathbf{U}$ & St & Uिa & ANES \\
\hline 2662 & $\operatorname{Tin} 113$ & $22 \pi 0$ & $\mathbf{u}$ & 0.6 & PCL & $A N$ \\
\hline 2032 & Tolume & $2 \sqrt[2]{20}$ & $\mathbf{u}$ & & TUEL & ANDS \\
\hline
\end{tabular}

WSRC-RP-99-00002 


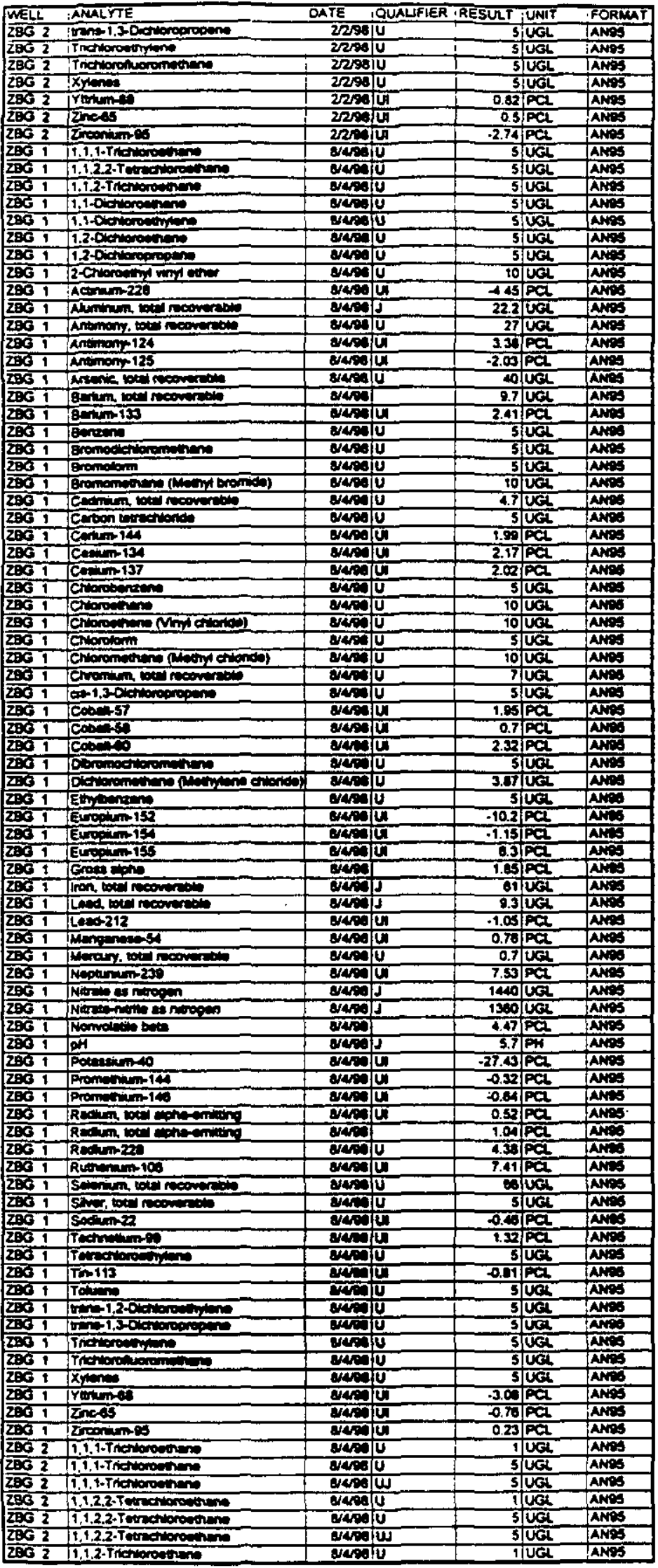




\begin{tabular}{|c|c|c|c|c|c|}
\hline WEL & ANALYTE & OATE & TQUALFER & IRESULT UUMT & FORMAT \\
\hline 2692 & 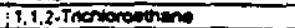 & vaso & 0 & Stü & ANOS \\
\hline $28 Q_{2}$ & $1,1,2$. Tnertopouthene & overs & U & suth & Ans \\
\hline$\angle E G 2$ & 1.1 .0 colorotions & VWSO & 10 & 10 & ANDS \\
\hline$Z B G 2$ & 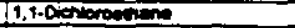 & rats & Iu & काणन & ANSB \\
\hline ZEG 2 & 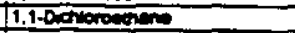 & vas & wu & SUa & AMSS \\
\hline 2802 & 1.1-Dion & owis & 0 & 1 uat & ANis \\
\hline 2802 & 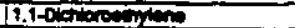 & vain & 0 & SUि & ANOS \\
\hline $2 E G 2$ & 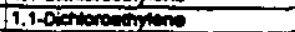 & Whin & w & SUUt & ANes \\
\hline$\angle E{ }_{2} 2$ & 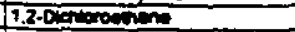 & Dais & U & IU⿺辶 & ANSS \\
\hline 2862 & 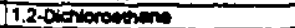 & क्रक्ज & u & ड़िक् & tangs \\
\hline 2602 & 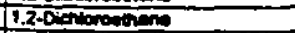 & gis & w & 5Uस & ANOS \\
\hline 2862 & 1.2-0iationopogen & Q4:0 & 0 & 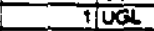 & ANS \\
\hline ZES 2 & 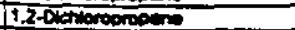 & 840 & 0 & SUTE & ANOS \\
\hline ZBG 2 & $11,2.0$ choropopen & प्रक्ष & wes & 5. UAL & ands \\
\hline ZEO 2 & 2-chorom vimat were & $\sin$ & 0 & s)uch & AN:S \\
\hline 2862 & 12-chorom vim etrer & rasis & 10 & rover & ANPS \\
\hline $280 \overline{2}$ & 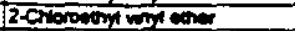 & $\sqrt{4 \pi}$ & ш & 100 & tans \\
\hline 2862 & Actinin 223 & Q4is: & un & $0.47 \mathrm{PC}$ & Anis \\
\hline 2802 & Activen 226 & 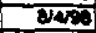 & U & $1.20 \mathrm{PC}$ & Ans \\
\hline 2862 & Aatinn-220 & Q14:0 & U1 & 11.19P्CL & Ans \\
\hline 2062 & Actinen-22] & 44 & U & $24.4 \mathrm{FC}$ & Ans \\
\hline 2862 & 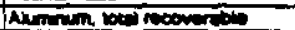 & avis & 2 & 12.1 Ua & ANaS \\
\hline 2862 & 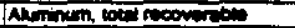 & Q 40 & IJ & 18.9ua & Axis \\
\hline 2002 & 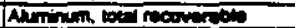 & Wक्ज & J & 4.7UG & AN: \\
\hline 2862 & 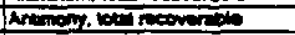 & vas & 0 & $0.2)$ & Ans \\
\hline $2 \overline{E G ~} 2$ & 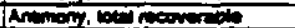 & QWW & U & 27) & ANS \\
\hline$\overline{2 B C 2}$ & Andinest 124 & Dese & UT & $-2.04 \overline{P C}$ & $A N=6$ \\
\hline 2852 & Ansinom-126 & arin & 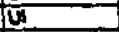 & D.MPC & Ax: \\
\hline 2002 & Ansinesing 124 & 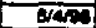 & ti & $3.57 / P C$ & ans \\
\hline $20 \overline{2}$ & Aromontizs & Med & $\omega$ & .2 .00 & $\sqrt{\operatorname{Nos}}$ \\
\hline 292 & aximein 1225 & wen & vis & -1.9fóa & Awes \\
\hline 2062 & Aromensias & Q⿻日木木 & U & $.1 .02 P C$ & and \\
\hline 2862 & Ardinow 123 & मavio & II & $1.82 P C$ & Nos \\
\hline 2062 & 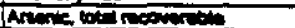 & 14: & जu & अणुड & ats \\
\hline 2062 & 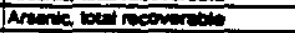 & Was & U & $40 \bar{a}$ & Ans \\
\hline 2002 & Betum wet neopertis & लक & & 5. Vर्द & Ans \\
\hline 2062 & 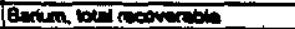 & pase & & 64U & ans \\
\hline 782 & 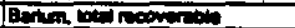 & Wi: & & 5.3400 & ANS \\
\hline 282 & $8=1+m i 3$ & Wis & Uि & $6.42 \mathrm{FC}$ & ans \\
\hline 2062 & Bentis3 & पर्या & $\mathbf{Q}$ & $431 P a$ & and \\
\hline 2002 & Banding & क्रो & U & $0.59 / \overline{C 2}$ & NWS \\
\hline 2002 & Basion & WN & U & TाUद & $A+3$ \\
\hline 262 & Berom & PLis & to & 5.0at & Nins \\
\hline 2802 & Bentins & Tas & w & 5) & ANS \\
\hline 2102 & aromodo forden & and & to & 10द & Ans \\
\hline 2002 & Econocion & पavis & $\overline{0}$ & 5 जिa & ALCS \\
\hline 2002 & 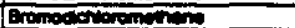 & 14 & $\boldsymbol{\omega}$ & 5ीर्: & ANC \\
\hline 2002 & Exonesom & awis & $\bar{v}$ & tua & ANB \\
\hline 2002 & Bromolorm & अक्त & U & SUिa & Ans \\
\hline 2002 & Erometion & Q6in & w & Stua & Nas \\
\hline ZEG 2 & 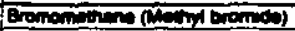 & Q45: & $\mathbf{U}$ & Tivat & ANi3 \\
\hline 2002 & 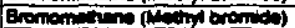 & बक्त & u & 10|va & ANDS \\
\hline 2862 & 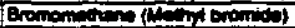 & 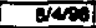 & एक & 10i्a & ANS \\
\hline$\overline{4062}$ & 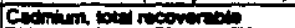 & क्लि & U & Tuet & 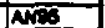 \\
\hline 2802 & 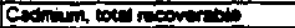 & ब्रक्ष & $\mathbf{U}$ & 4.7ण्ड & AN \\
\hline 2802 & 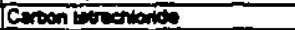 & स्तis & to & TUE & ANis \\
\hline 2002 & 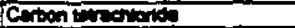 & श्रा & 0 & 5 पिल्य & $\sqrt{A M B}$ \\
\hline 2002 & Corten trations & Q4wit & W & 5ीज्र & Nos \\
\hline 2802 & Contring & $\ln$ & $\mathbf{u}$ & $-3.03 / P C$ & An: \\
\hline 2802 & Coniminia & 90140 & $\mathbf{u}$ & $2.63 \mathrm{FC}$ & AN \\
\hline 2802 & Cortumist & Q4ivin & $\mathbf{u}$ & 7.47 FC & AWS \\
\hline 202 & Continia & 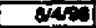 & $\mathbf{U}$ & $7 . 5 \longdiv { P C }$ & $A=$ \\
\hline 2062 & $\cos \ln 134$ & स्थ & $\mathbf{U}$ & $.1,20 \mid \overline{C A}$ & NSS \\
\hline 202 & Cenim 134 & QMen & $\mathbf{u}$ & $0 . \overline{2} \overline{C a}$ & AN? \\
\hline 296 & Conten-134 & 8 & IT & $3.44 \mathrm{DC}$ & ANis \\
\hline 2063 & Cenimis & QNa & $\mathbf{W}$ & $0.002 \mathrm{FC}$ & ANS \\
\hline 2002 & $c_{0}=137$ & प्र & $\mathbf{U}$ & $0.5 \% 7, \mathrm{FC}$ & AN \\
\hline दEQ 2 & Co:137 137 & 80 & W & $1 . \mathrm{DFC}$ & aW \\
\hline 202 & Centin 137 & yin & $\mathbf{U}$ & $2.15 F$ & $\operatorname{An}=3$ \\
\hline 2802 & Canding 137 & $\sin$ & $\mathbf{n}$ & $2.97 \times C$ & ANO \\
\hline$\angle B O 2$ & Cropontes & 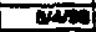 & ए\zh14 & गिएक & 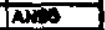 \\
\hline ZEO & 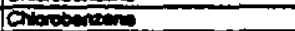 & Wra & $\mathbf{u}$ & SUE⿱ & Ans \\
\hline 23 & Criopingeres & arat & $\boldsymbol{\omega}$ & SUal & $A_{1}=3$ \\
\hline 2062 & Crovenom & vin & $\mathbf{v}$ & गUa & $N=3$ \\
\hline 2062 & cripomine & 20 & $\bar{U}$ & 10:4e & $\sqrt{2} 3$ \\
\hline 2002 & Cromonion & 8 & W & 100्2 & ANS \\
\hline 2862 & 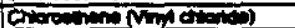 & 80 & U & Tण्2 & ANS \\
\hline 2002 & 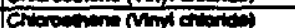 & अ० & 0 & $10.0 \mathrm{x}$ & Ants \\
\hline 2062 & 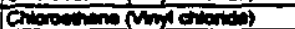 & Wwis & W & 10ण्य & AN: \\
\hline 2002 & Otrorotom & ros & u & 1) & 3 ANG \\
\hline 262 & Crionotom & 140 & $\bar{U}$ & Stuat & ANo \\
\hline 2962 & Chorotom & Meres & w & S|UE & ANOS \\
\hline 2062 & 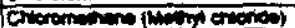 & Varis & U & Túa & Ans \\
\hline 2002 & 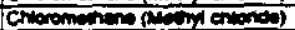 & Vesiv & u & 10.üL & ANDS \\
\hline 2062 & 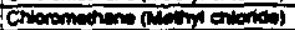 & vesin & u & $100 \mathrm{c}$ & AnOS \\
\hline 2932 & 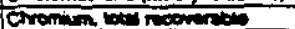 & vart & 5 & $1.0 \mathrm{UL}$ & Ans \\
\hline 2802 & 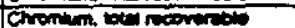 & प्रक्य & Iu & 1.7 uिa & AMS \\
\hline 2062 & 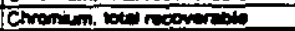 & Wos: & ț & 7 पua & ANS \\
\hline
\end{tabular}

WSRC-RP-99-00002 


\begin{tabular}{|c|c|c|c|c|c|}
\hline MEC & ANALITE & OATE & QUAUIFER & IRESULT UNAT & Forma? \\
\hline 2962 & 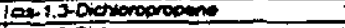 & 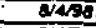 & IU & THQ & ANOS \\
\hline $2 \mathrm{EG} 2$ & 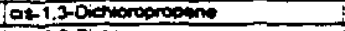 & 240 & IU & S:UGL & ANOS \\
\hline ZBG 2 & 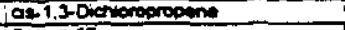 & vase & yus & 5ाUG & ANOS \\
\hline 2EG 2 & 1Cober. 57 & garag & ivi & $0.101 P C 1$ & ANOS \\
\hline $2 E G 2$ & Copon.57 & 0 & UI & $0.6 \% \mathrm{FL}$ & ANes \\
\hline EEG 2 & $\cos =57$ & $8 \times \sqrt{20}$ & is & $0.755 P C$ & ANSS \\
\hline ZBG 2 & $006-57$ & aris & Tu & 1.52) & TANOS \\
\hline ZBO 2 & $\cos =5$ & 840 & U. & $0.02 / \mathrm{FCL}$ & ANOS \\
\hline ZEG 2 & $\cos =5$ & 240 & IU & $1.02 \mathrm{PC}$ & ANS \\
\hline 2002 & $\cos 0$ & vas & IU. & $1.5 \mathrm{P} P \mathrm{PC}$ & ANOS \\
\hline 2062 & $\cos \theta-\infty$ & Warts & UT & .2 .80 & ANos \\
\hline 2002 & $\cos =0$ & $8 a^{3}$ & U: & $0.33: \mathrm{Ca}$ & ANOS \\
\hline 2862 & $\cos =-\infty$ & owis & Uin & 1.3/AC & ANAS \\
\hline 2BG 2 & $\cos =100$ & Das & W & 1.71/AC & $\sqrt{A N O S}$ \\
\hline $2 E G 2$ & 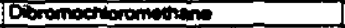 & van & to & 1 जि & ANDS \\
\hline 2062 & 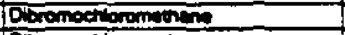 & yanas & U & Stua & ANBS \\
\hline 2862 & Dosomoctionomenense & Maria & iu & Swa & ANES \\
\hline 2802 & 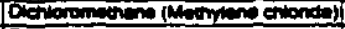 & Wक & U & 4.02 Ua & ANES \\
\hline 2002 & 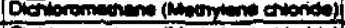 & s/ang & U & 1) & ANis \\
\hline 2062 & 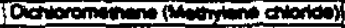 & Qwat & Uu & 3.9) va & ANS \\
\hline ZEG 2 & Etmpentingen & Thes & 0 & 1 Uat & ANes \\
\hline ZEG 2 & Expromate & Q V4:0 & IU & 5ण्a & ANES \\
\hline 2BC 2 & Etmionsions & yen & W & SIVE: & ANS \\
\hline 28,2 & Everintis2 & प्रas & U. & .17 .53 [PC & ANOS \\
\hline 2862 & 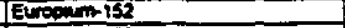 & vasi] & $\mathbf{W}$ & $-2.77 P C$ & ANS \\
\hline ZEG 2 & Eurapin-152 & vasis & $\mathbf{U}$ & $3.1010 \mathrm{C}$ & ANAS \\
\hline $282_{2}$ & Euroginimis & P4in & U & 12.31EC & ANS \\
\hline 2602 & Eurogimis & पena & U & $.5 .52 \sqrt{20}$ & ANiS \\
\hline 2862 & Europien-154 & Whan & Uు & $.1 .40 \mathrm{C}$ & ANes \\
\hline 2862 & Evogem is & WWa & $\bar{\omega}$ & 3.0. & ANOS \\
\hline 2802 & Eveonimisa & Bara & UI & $0.021 P C$ & ANes \\
\hline 2962 & Eurooumiss & vis & $\underline{\omega}$ & $.1 .52] \overline{P C}$ & AN: \\
\hline 2002 & Ergoming & Nato & U⿺ & $9.21 \sqrt{\mathrm{PC}}$ & IAK:S \\
\hline 2062 & Exropinmintiss & प्रक्य & $\bar{U}$ & $2.87 \sqrt{\mathrm{PC}}$ & ANES \\
\hline ZEO2 & Europen-153 & Qwa & IU & $2.3[\bar{C}$ & Non \\
\hline 2002 & 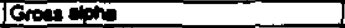 & 940 & u & $0.321 P C$ & ANBS \\
\hline ZES 2 & Gros: whe & 340 & Un & $0.525 \mathrm{PEC}$ & $A N{ }^{\infty}$ \\
\hline 2062 & Groes enen & vars & & 0.775 PC: & ANES \\
\hline 2602 & Groes ant & exin & & $0.03 / \overline{P C}$ & ANis \\
\hline ZES & Goasente & प्र400 & & $1 . 2 2 \longdiv { \mathrm { PC } }$ & Taris \\
\hline 202 & Iron wail heoveren & 940 & J & $24.0 \overline{\mathrm{Ua}}$ & NAS \\
\hline 2032 & 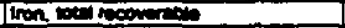 & 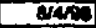 & 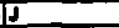 & Q4.3णि & ANis \\
\hline 202 & 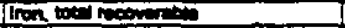 & 0 & & 51010a & AN:S \\
\hline 2802 & 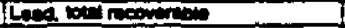 & 040 & $\sqrt{3}$ & $0.07 / \bar{a}$ & AN: \\
\hline 260 & 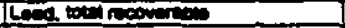 & 0,0 & $\omega$ & $97 \overline{04}$ & AN:S \\
\hline 2832 & 160.212 & Val & W & $.1 .91 / 2$ & ans \\
\hline 2002 & Lene212 & Q940 & $\bar{u}$ & S.41FC & ANS \\
\hline 2802 & Lex212 & ren & $\overline{I N}$ & $7.07 \overline{P C}$ & ANG \\
\hline 2862 & $\sqrt{60+2272}$ & 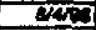 & & $100 . \overline{F C}$ & M:S \\
\hline 2802 & Non & Qwis & $\mathbf{N}$ & $.2 .0 \overline{F C}$ & AN: \\
\hline 2602 & 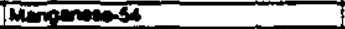 & gat & $\omega$ & $. 1 . 2 6 \longdiv { P C }$ & AN:S \\
\hline 2632 & 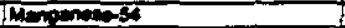 & $\sqrt{4}$ & U & $0.72 F C$ & ance \\
\hline 202 & 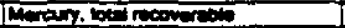 & vation & $\mathbf{0}$ & 0.7 Ua & ANiS \\
\hline 2002 & 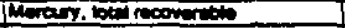 & 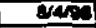 & & 0.7 Ua & Nes \\
\hline 2802 & 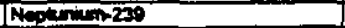 & Qvas & W & $4 3 . 6 1 \longdiv { P C }$ & Ans \\
\hline $20 \overline{2}$ & Noparium 230 & Ware & $\bar{u}$ & $-12.00 / \bar{C}$ & ANis \\
\hline 2802 & Nopennim-230 & Yais & $\mathbf{U}$ & $-1.07 \widetilde{F C}$ & AN:S \\
\hline 2652 & Nitrenes nivopin & 8 & Is & 570 Ua & NAS \\
\hline 2632 & Nis: an norm & 840 & IJ & 57DUCL & ANBS \\
\hline 2062 & $N_{M}$ & 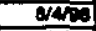 & 5 & 53006 & ANOS \\
\hline 282 & 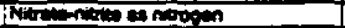 & Qvin & & $500 / \overline{6}$ & ANis \\
\hline 2062 & Now en nomen & Non & (0) & Solva & ANES \\
\hline 2862 & 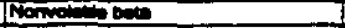 & $\mathbf{W 4}$ & ए0 & $900 \mathrm{Fa}$ & ANS \\
\hline 2002 & 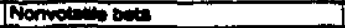 & एकin & 0 & $17.87 \overline{F C}$ & ANC \\
\hline 2602 & Nowo: & 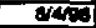 & U & $0.81 \mathrm{FC}$ & JA:S \\
\hline $20 \overline{32}$ & Nomolom to & 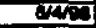 & एu & $1 . 3 \longdiv { \overline { Q C } }$ & AN:3 \\
\hline 2862 & Nomomingen & प्रक्ष & & 1.45 pct & AN \\
\hline 2802 & IPH & $0 \pi 0$ & 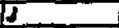 & $5.37 \mathrm{PH}$ & JANS \\
\hline$\angle A B 2$ & pan & प्रक्ष & 5 & 5.30 $\mathrm{PH}$ & ANiS \\
\hline ZEG 2 & Tpi & 20 & 5 & $5.51 \mathrm{PH}$ & ANS \\
\hline 2802 & ton & wa & 3 & $5.55 \mathrm{PH}$ & ANis \\
\hline ZEG 2 & Potengen & 0 & D & .14 .719 & ANeS \\
\hline 2862 & Pot & $8 x=$ & U & $2.451 \overline{P C}$ & AN \\
\hline 2802 & Poten: & प्रs: & $E$ & $32.6 \mathrm{FC}$ & ANB \\
\hline 2932 & PQE & man & & $39.52[\overline{C E}$ & AN: \\
\hline 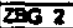 & Alominimita & 00 & 保 & $7.62 \sqrt{P C}$ & Anis \\
\hline 2002 & Promenteris & 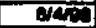 & U & $.1 .9 \mathrm{FC}$ & ANS \\
\hline 2002 & Promenthentan & $2 \times$ & $\mathbf{U}$ & $0.01 / \bar{C}$ & ANis \\
\hline 28 & 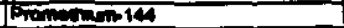 & Wat & 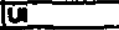 & $0.81 P C$ & ANSS \\
\hline 2962 & Prominimise & $\mathrm{win}$ & U & $- 3 . 2 \longdiv { F C }$ & ANes \\
\hline 2062 & 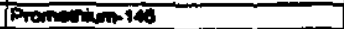 & प्रaro & U & $-1.5 \mathrm{TAC}$ & AN:S \\
\hline ZEG 2 & porominim-146 & 200 & U & $1.72, \overline{P C}$ & Ans \\
\hline ZEG 2 & Promeniminis & risa & un & $3.5 \mid F \mathrm{C}$ & ANis \\
\hline $28 \overline{2}$ & 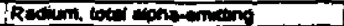 & 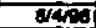 & IजI & $0.10 \rho \alpha$ & ANES \\
\hline सEG 2 & 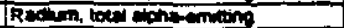 & Q1400 & जi & $0.21 / \overline{P C}$ & ANOS \\
\hline ZBG 2 & 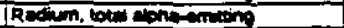 & 840 & $\bar{v}$ & $0.3 \sqrt[P C]{ }$ & ANAS \\
\hline EAG 1 & $\operatorname{lin} 20$ & 20 & & O.SOPC & ANCS \\
\hline ZEG 2 & Redingen 230 & 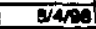 & UI & $0.181 P C 1$ & ANes \\
\hline
\end{tabular}

WSRC-RP-99-00002

I-16 


\begin{tabular}{|c|c|c|c|c|c|c|}
\hline MELLI & TANACTE & DATE & DOULGFIE & TRESULT & एWNIT & Fonimar \\
\hline$\angle B G 2$ & $R=0 \ln -20$ & QWN & गएज & 0.34 & APCL & IANOS \\
\hline 2002 & Recing 232 & Whe & iv & 0.43 & त्वC & TANSS \\
\hline $2 B G_{2}$ & iRecom-230 & Whe & & 9.48 & $\overline{A C L}$ & ANOS \\
\hline 2862 & Recion 220 & gine & & 1.67 & 7) & ANos \\
\hline$\overline{20,2}$ & $R=0 m 20$ & Wis & $\bar{U}$ & -0.33 & $3 \longdiv { P C L }$ & ANeS \\
\hline $2 B 6 \overline{2}$ & Recin-2a & $8 \omega^{2}$ & it & 0.55 & StPCL & Anis \\
\hline 2062 & Recisen & $\sqrt{2}$ & 0 & 0.037 & $7 \longdiv { A C }$ & $A N B$ \\
\hline $2 \overline{62}$ & Recinzan & ent & Iu & 2.39 & $\sqrt{A C 2}$ & ANSS \\
\hline 2902 & Renenas & $8 \times$ & & 0.067 & $\sqrt{\mathrm{FCL}}$ & ANOS \\
\hline $280 \overline{2}$ & Ricrician 106 & an & Uu & 28.70 & $\overline{P C L}$ & ANOS \\
\hline$\overline{2 E Q 2}$ & RLOTing & बक्षि & U & .16 .75 & $5 \mid F C$ & ANDS \\
\hline 2802 & RLtiminan 100 & बiv & U. & .15 .55 & $5 \overline{P C}$ & ANOS \\
\hline $28 Z_{2}$ & RLOTn=10 & $\sqrt{20}$ & iv & 9.03 & IPCI & ANDS \\
\hline 2602 & 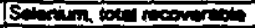 & varin & एu & & SUG & TANDS \\
\hline 2062 & 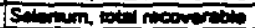 & $8 / \sqrt{2}$ & 0 & 8 & tug & ANES \\
\hline ZEG 2 & 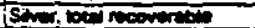 & $\operatorname{los}$ & Iu & 0.54 & U⿺辶 & ANDS \\
\hline 282 & 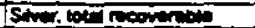 & $\operatorname{ann}$ & Uú & & पिa्र & ANAS \\
\hline 2802 & 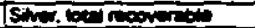 & Q6/2 & itu & $\mathbf{5}$ & 5 जual & ANBS \\
\hline$\overline{\angle B 2}$ & $50 \ln 22$ & 84 & $\bar{\omega}$ & -1.82 & $3 \longdiv { P C L }$ & ANOS \\
\hline 2832 & sotimna & $\sqrt{2}$ & $\vec{U}$ & 0.54 & 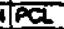 & ANBS \\
\hline 2062 & solumz & 8क्रि & $\bar{u}$ & 1.30 & DPa & ANis \\
\hline 2962 & sociuma & vain & IU & 2.46 & $P C$ & AN:S \\
\hline 2062 & Tortinginte & avin & $\bar{u}$ & .2 .2 & शीPC & ANAS \\
\hline 2602 & Tecturentes & vin & IU & .1 .01 & iीC & ANAS \\
\hline 202 & Tectinisinge & 80 & $\bar{u}$ & -0.73 & $\sqrt{P C L}$ & $\sqrt{1 \times 6}$ \\
\hline$2 \longdiv { 6 2 }$ & Treosingen & vivi & w & 0.503 & BAC & Ancs \\
\hline 2002 & 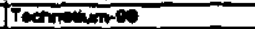 & 940 & wis & 3.5 & $\overline{P a}$ & $\sqrt{A N B}$ \\
\hline 2062 & The & $\sqrt{20}$ & Tu & & iण्a & ANS \\
\hline 2002 & 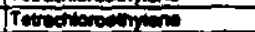 & $\sqrt{4}$ & 0 & 5 & SUिद & ANS \\
\hline 2002 & 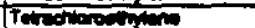 & M4: & Wक & & 5 viat & $A M B$ \\
\hline 202 & $\operatorname{Tin} 113$ & $\sin$ & Tu & .3 .30 & $\overrightarrow{P C C}$ & $\sqrt{1 \times 3}$ \\
\hline 2002 & $\sqrt{17513}$ & क्षis & U & 0.04 & $\overrightarrow{P a}$ & $A N B$ \\
\hline 202 & $T=113$ & $8 \sqrt{5}$ & $\overline{0}$ & 0.20 & DPC & ANis \\
\hline 2002 & Tokene & 20in & tu & & tuat & Aris \\
\hline 2002 & Totenese & $\sqrt{\infty}$ & $\sqrt{0}$ & 5 & S.णूर & IAN: \\
\hline 2062 & Tonewe & QW: & क्य & & 5 प्रद & ANS \\
\hline$\overline{82}$ & 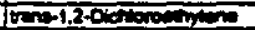 & gw: & U & 1 & 1.uat & AMBS \\
\hline 2862 & 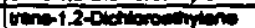 & Wht & u & 5 & जuat & ANDS \\
\hline 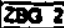 & 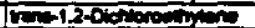 & Wकी & w & $\overline{5}$ & 5ॉणिद् & $\overline{A N B}$ \\
\hline 2832 & 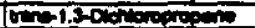 & प्रका & U & & Tिa & $\sqrt{A N D S}$ \\
\hline 2032 & tol 1.300100 & ave & 0 & 5 & 5णिa & AN:S \\
\hline 2032 & $(5=1,300000$ & WW: & IU & & stü & ANIS \\
\hline 2032 & 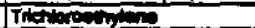 & पwa & 10 & 1 & 10द & $1 \mathrm{NOB}$ \\
\hline 26032 & 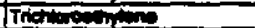 & क्य. & 10 & & काणि & Ancs \\
\hline 2002 & Therimone & बivi & w & & काष्ट & ANC \\
\hline 2802 & 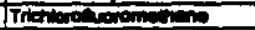 & 840. & 0 & & tual & Ants \\
\hline 2802 & 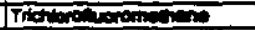 & $\sqrt{2}$ & U & 3 & Sप्रे & ANBS \\
\hline 2002 & 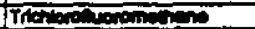 & $8 / 4$ & Ww & 3 & $3 \longdiv { 0 a }$ & ANis \\
\hline 2002 & $x=$ & 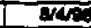 & एu & & 5 & Anis \\
\hline 2862 & $x$ & $\sqrt{4}$ & w & & Stual & axis \\
\hline 202 & Vton & $\sqrt{4 \sigma^{2}}$ & T & 3.20 & ACL & ANSS \\
\hline ZEG 2 & Yatenes & क्षiv & U & .3 .03 & उन्टर & AMS \\
\hline 203 & rotenes & owin & Tu & -9.44 & A्Ct & ANS \\
\hline 2862 & rotento & $\operatorname{las}$ & $\bar{\omega}$ & 0.0 & OFCL & AN \\
\hline $2 \mathrm{EOS}$ & zoness & vasis & iv & 6.94 & $\sqrt{A C}$ & $\overline{\operatorname{ANC} B}$ \\
\hline 2662 & zinos & arin & U & 6.70 & $P C$ & ANDS \\
\hline 2032 & Znces & So: & U & .2 .00 & $\overline{P C L}$ & and \\
\hline 2002 & Enos & visi & in & 0.79 & $A C$ & ANES \\
\hline 2002 & Zconthes & $84 \%$ & tus & .9 .53 & 3 & $\longdiv { A N C S }$ \\
\hline 2003 & Zrooninos & viv & 10 & 0.75 & $\overline{F C}$ & ANES \\
\hline 2862 & zoronges & 8 & U & 8.50 & OPC & ANSS \\
\hline
\end{tabular}

WSRC-RP-99-00002 
APPENDIX II

FIELD DATA

II-1 


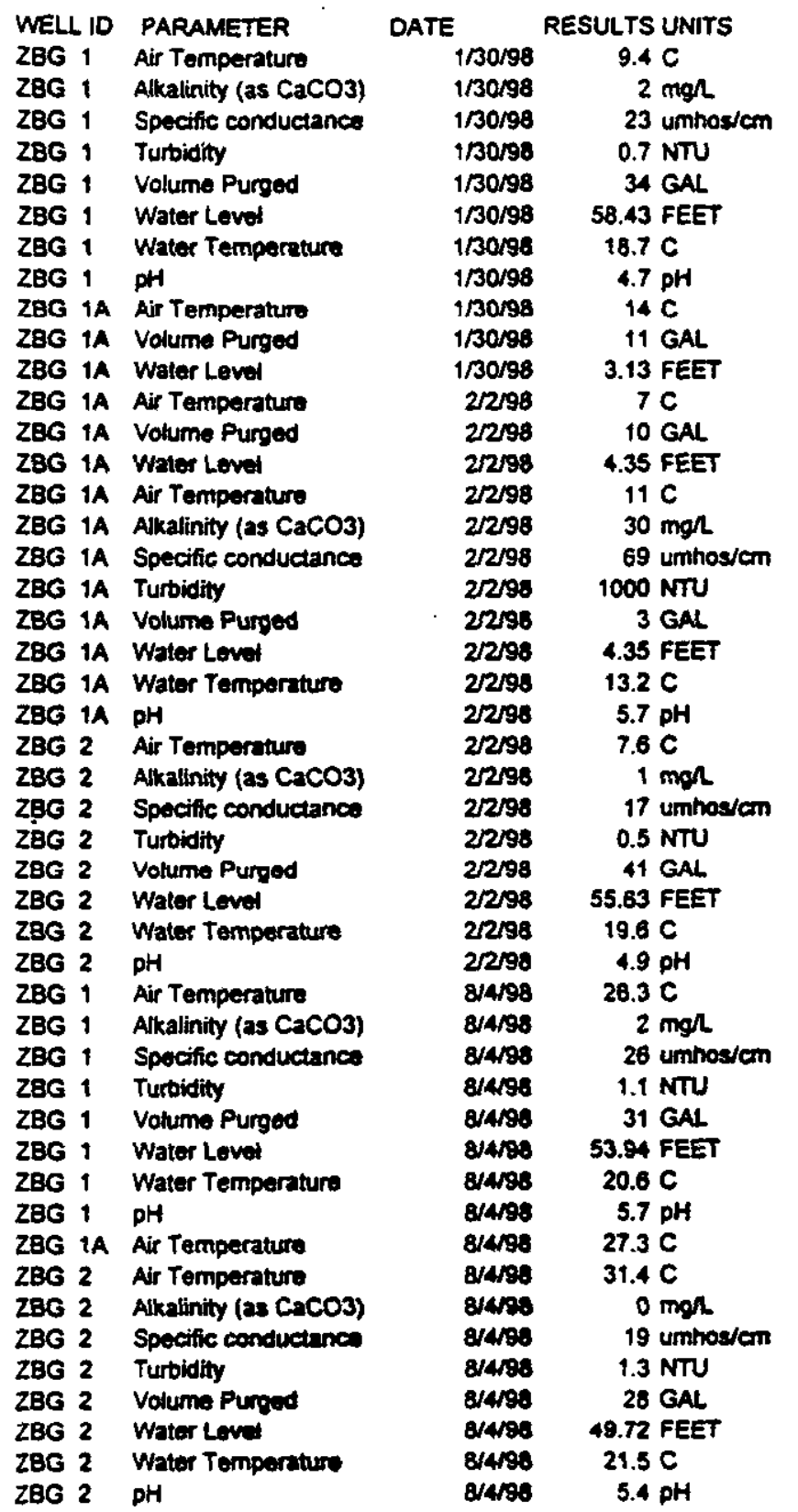


APPENDIX III

QUALIFIERS 
(Blank)

E

I

J

L

M

R

T

U

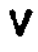

$Y$

1

2

3

Data are not qualified. Numbers should be interpreted exactly as reported. The detected result is between the sample-specific EQL and the method detection fimit.

The value in the result field is the instrument reading, not the sample quantitation limit. Always used with the resuth qualifier $U$.

Value is estimated because quantitation in the sample or in associated quality control samples did not meot specifications.

Value is off-scale high. The actual value is not known but is known to be greater than the value shown.

Presence of the analyte is verified butnot quantified.

Result was rejected because performance requirements in the sample analysis or associated quality control analyses were not met.

Analyte was not detected; if present, it was below the criteria for detection.

Material analyzed for but not detected. Analyical result reported is less than the sample quantitation limit.

Analyte was detected in an associated method blank.

Result was obtained from an unpreserved or improperty preserved sample. Data may not be accurate.

Result may be an underestimation of the true value due to analytical bias.

Result may be an overestimation of the thue value due to analytical bias.

The associated result may be of poor precision (high variability) due to analytical bias.

Note: These are onty some of the qualifiers present in the database. Al modifiers associated with the data are publishod in tho reach tables of EPD/EMS' quarterty groundwater monitoring reports, the official repository of the date. 